\title{
FREQUENCY OF SOLAR-LIKE SYSTEMS AND OF ICE AND GAS GIANTS BEYOND THE SNOW LINE FROM HIGH-MAGNIFICATION MICROLENSING EVENTS IN 2005-2008
}

\author{
A. Gould ${ }^{1,2}$, Subo Dong ${ }^{1,3,82}$, B. S. Gaudi ${ }^{1,2}$, A. Udalski ${ }^{4,5}$, I. A. Bond ${ }^{6,7}$, J. Greenhill ${ }^{8,9}$, R. A. Street $^{10,11,83}$, \\ M. DominiK ${ }^{8,12,13,83,84}$, T. Sumi ${ }^{6,14}$, M. K. SzYMAŃski ${ }^{4,5}$, C. HaN ${ }^{1,15}$, \\ AND
}

W. Allen ${ }^{16}$, G. Bolt ${ }^{17}$, M. Bos ${ }^{18}$, G. W. Christie ${ }^{19}$, D. L. DePoy ${ }^{20}$, J. Drummond $^{21}$, J. D. EASTMAN $^{2}$, A. GAl-YAM $^{22}$,

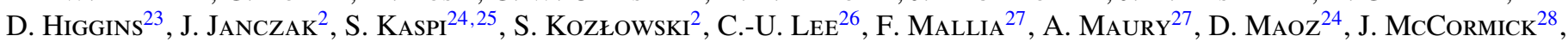
L. A. G. Monard ${ }^{29}$, D. Moorhouse ${ }^{30}$, N. Morgan ${ }^{2}$, T. Natusch ${ }^{31}$, E. O. OfeK ${ }^{32,85}$, B.-G. Park ${ }^{26}$, R. W. Pogge $^{2}$, D. Polishook ${ }^{24}$, R. Santallo ${ }^{33}$, A. Shrorer ${ }^{24}$, O. Spector ${ }^{24}$, G. Thornley ${ }^{30}$, J. C. YeE $^{2}$

(The $\mu$ FUN COLlaboration),

AND

M. Kubiak ${ }^{5}$, G. Pietrzyński ${ }^{5,34}$, I. Soszyński ${ }^{5}$, O. SzewCZyK ${ }^{34}$, Ë. Wyrzykowski $^{35}$, K. UlaczyK $^{5}$, R. Poleski $^{5}$

(The OGLE Collaboration),

AND

F. Abe ${ }^{14}$, D. P. Bennetr ${ }^{8,36}$, C. S. Botzler ${ }^{37}$, D. Douchin ${ }^{37}$, M. Freeman ${ }^{37}$, A. Fukui ${ }^{14}$, K. Furusawa ${ }^{14}$, J. B. Hearnshaw ${ }^{38}$, S. Hosaka ${ }^{14}$, Y. Itow ${ }^{14}$, K. Kamiya ${ }^{14}$, P. M. Kilmartin ${ }^{39}$, A. Korpela ${ }^{40}$, W. Lin ${ }^{7}$, C. H. Ling ${ }^{7}$, S. MaKita $^{14}$, K. Masuda ${ }^{14}$,

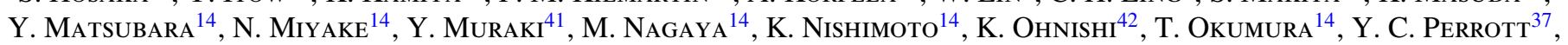
L. Philpott ${ }^{37}$, N. Rattenbury ${ }^{37,83}$, To. Saito ${ }^{43}$, T. Sako $^{14}$, D. J. Sullivan ${ }^{40}$, W. L. Sweatman ${ }^{7}$, P. J. Tristram $^{39}$, E. VON SEGGERN ${ }^{37}$, P. C. M. YOCK ${ }^{37}$

(The MOA Collaboration),

AND

M. Albrow ${ }^{38}$, V. Batista ${ }^{44}$, J. P. Beaulied ${ }^{44}$, S. Brillant ${ }^{45}$, J. Caldwell ${ }^{46}$, J. J. Calitz ${ }^{47}$, A. Cassan ${ }^{44}$, A. Cole $^{9}$, K. Cook $^{48}$, C. Coutures ${ }^{49}$, S. Dieters ${ }^{44}$, D. Dominis Prester ${ }^{50}$, J. Donatowicz $^{51}$, P. Fouqué ${ }^{52}$, K. Hill $^{9}$, M. HofFMan ${ }^{47}$, F. Jablonski $^{53}$, S. R. Kane ${ }^{54}$, N. Kains ${ }^{13,83}$, D. Kubas ${ }^{45}$, J.-B. Marquette ${ }^{44}$, R. Martin ${ }^{55}$, E. Martioli ${ }^{53}$, P. MeintJes ${ }^{47}$, J. MenZies ${ }^{56}$, E. Pedretti ${ }^{13}$, K. Pollard ${ }^{38}$, K. C. Sahu ${ }^{57}$, C. Vinter $^{58}$, J. Wambsganss ${ }^{12,59}$, R. Watson ${ }^{9}$, A. Williams ${ }^{55}$, M. Zub ${ }^{59,60}$

(The Planet Collaboration),

AND

A. Allan ${ }^{61}$, M. F. Bode ${ }^{62}$, D. M. Bramich ${ }^{8,63}$, M. J. Burgdorf ${ }^{12,64,65}$, N. Clay $^{62}$, S. FraSER $^{62}$, E. HAWKINS $^{10}$, K. Horne $^{8,13}$,

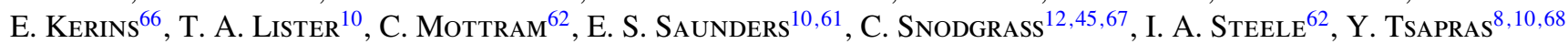

(The Robonet Collaboration),

AND

U. G. Jørgensen ${ }^{8,58,69}$, T. Anguita ${ }^{59,70}$, V. Bozza ${ }^{71,72,73}$, S. Calchi Novati ${ }^{71,72,73}$, K. HarpsøE ${ }^{58}$, T. C. Hinse ${ }^{58,74}$,

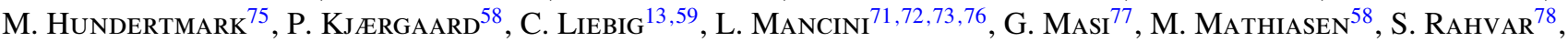

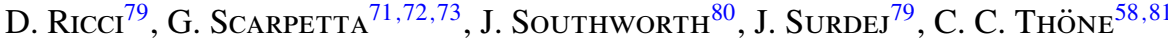

(The MiNDSTEp Consortium)

${ }^{1}$ Microlensing Follow Up Network ( $\mu$ FUN)

2 Department of Astronomy, Ohio State University, 140 W. 18th Ave., Columbus, OH 43210, USA; gould@ astronomy.ohio-state.edu, gaudi@astronomy.ohio-state.edu, jdeast@astronomy.ohio-state.edu, jyee@ astronomy.ohio-state.edu, pogge@astronomy.ohio-state.edu, simkoz@astronomy.ohio-state.edu, nick.morgan@alum.mit.edu

${ }^{3}$ Institute for Advanced Study, Einstein Drive, Princeton, NJ 08540, USA; dong @ias.edu

${ }^{4}$ Optical Gravitational Lens Experiment (OGLE)

${ }^{5}$ Warsaw University Observatory, Al. Ujazdowskie 4, 00-478 Warszawa, Poland; udalski@ astrouw.edu.pl, msz@ astrouw.edu.pl, mk@ astrouw.edu.pl, pietrzyn@astrouw.edu.pl, soszynsk@astrouw.edu.pl,kulaczyk@astrouw.edu.pl,rpoleski@astrouw.edu.pl

${ }^{6}$ Microlensing Observations in Astrophysics (MOA)

${ }^{7}$ Institute of Information and Mathematical Sciences, Massey University, Private Bag 102-904, North Shore Mail Centre, Auckland, New Zealand; i.a.bond@massey.ac.nz,1.skuljan@massey.ac.nz,w.lin@massey.ac.nz,c.h.ling@massey.ac.nz,w.sweatman@massey.ac.nz

${ }^{8}$ Probing Lensing Anomalies NETwork (PLANET)

${ }^{9}$ University of Tasmania, School of Mathematics and Physics, Private Bag 37, GPO Hobart, Tas 7001, Australia; John.Greenhill@utas.edu.au, Andrew.Cole@utas.edu.au

${ }^{10}$ Las Cumbres Observatory Global Telescope network, 6740 Cortona Drive, suite 102, Goleta, CA 93117, USA

${ }^{11}$ Department of Physics, Broida Hall, University of California, Santa Barbara, CA 93106-9530, USA

${ }^{12}$ Microlensing Network for the Detection of Small Terrestrial Exoplanets (MiNDSTEp)

${ }^{13}$ SUPA School of Physics and Astronomy, University of St. Andrews, KY16 9SS, UK; md35@ st-andrews.ac.uk, nk87@ st-andrews.ac.uk, ep41@ @t-andrews.ac.uk, kdh1@st-andrews.ac.uk

${ }^{14}$ Solar-Terrestrial Environment Laboratory, Nagoya University, Nagoya 464-8601, Japan; sumi@ stelab.nagoya-u.ac.jp, abe@ stelab.nagoya-u.ac.jp, afukui@stelab.nagoya-u.ac.jp, furusawa@ stelab.nagoya-u.ac.jp, itow@stelab.nagoya-u.ac.jp, kkamiya@ stelab.nagoya-u.ac.jp, kmasuda@ stelab.nagoya-u.ac.jp, ymatsu@stelab.nagoya-u.ac.jp,nmiyake@stelab.nagoya-u.ac.jp, mnagaya@ stelab.nagoya-u.ac.jp, okumurat@ stelab.nagoya-u.ac.jp, sako@stelab.nagoya-u.ac.jp

${ }^{15}$ Department of Physics, Institute for Basic Science Research, Chungbuk National University, Chongju 361-763, Korea; cheongho@ astroph.chungbuk.ac.kr ${ }^{16}$ Vintage Lane Observatory, Blenheim, New Zealand; whallen@xtra.co.nz ${ }^{17}$ Craigie Observatory, Perth, Australia; gbolt@iinet.net.au

${ }^{18}$ Molehill Astronomical Observatory, Auckland, New Zealand; molehill@ihug.co.nz

${ }_{19}$ Auckland Observatory, Auckland, New Zealand; gwchristie@ christie.org.nz 
${ }^{20}$ Department of Physics and Astronomy, Texas A\&M University, College Station, TX, USA; depoy@physics.tamu.edu

${ }^{21}$ Possum Observatory, Patutahi, New Zealand; john_drummond@xtra.co.nz

${ }^{22}$ Department of Particle Physics and Astrophysics, Weizmann Institute of Science, 76100 Rehovot, Israel; avishay.gal-yam@weizmann.ac.il

${ }^{23}$ Hunters Hill Observatory, Canberra, Australia; dhi67540@bigpond.net.au

${ }^{24}$ School of Physics and Astronomy and Wise Observatory, Tel-Aviv University, Tel-Aviv 69978, Israel; shai@wise.tau.ac.il, dani@wise.tau.ac.il, david@wise.tau.ac.il, shporer@wise.tau.ac.il,odedspec@wise.tau.ac.il ${ }^{25}$ Department of Physics, Technion, Haifa 32000, Israel

${ }^{26}$ Korea Astronomy and Space Science Institute, Daejon 305-348, Korea; leecu@kasi.re.kr, bgpark@kasi.re.kr

${ }^{27}$ Campo Catino Austral Observatory, San Pedro de Atacama, Chile; francomallia@campocatinobservatory.org, alain@spaceobs.com

${ }^{28}$ Farm Cove Observatory, Centre for Backyard Astrophysics, Pakuranga, Auckland, New Zealand; farmcoveobs@xtra.co.nz

${ }^{29}$ Bronberg Observatory, Centre for Backyard Astrophysics, Pretoria, South Africa; lagmonar@nmisa.org

30 Kumeu Observatory, Kumeu, New Zealand; acrux@orcon.net.nz, guy.thornley@gmail.com

${ }^{31}$ AUT University, Auckland, New Zealand; tim.natusch@aut.ac.nz

32 Palomar Observatory, CA, USA; eran@ astro.caltech.edu

${ }^{33}$ Southern Stars Observatory, Faaa, Tahiti, French Polynesia; obs930@ southernstars-observatory.org

${ }^{34}$ Departamento de Fisica, Universidad de Concepción, Casilla 160-C, Concepción, Chile; szewczyk @astro-udec.cl

${ }^{35}$ Institute of Astronomy, University of Cambridge, Madingley Road, CB3 0HA Cambridge, UK; wyrzykow@ast.cam.ac.uk

${ }^{36}$ Department of Physics, University of Notre Dame, 225 Nieuwland Science Hall, Notre Dame, IN 46556-5670, USA; bennett@nd.edu

${ }^{37}$ Department of Physics, University of Auckland, Private Bag 92019, Auckland, New Zealand; c.botzler@auckland.ac.nz, p.yock@auckland.ac.nz, yper006@aucklanduni.ac.nz

${ }^{38}$ Department of Physics and Astronomy, University of Canterbury, Private Bag 4800, Christchurch 8020, New Zealand

${ }^{39}$ Mt. John Observatory, P.O. Box 56, Lake Tekapo 8780, New Zealand

${ }^{40}$ School of Chemical and Physical Sciences, Victoria University, Wellington, New Zealand; a.korpela@niwa.co.nz, denis.sullivan@vuw.ac.nz

${ }^{41}$ Department of Physics, Konan University, Nishiokamoto 8-9-1, Kobe 658-8501, Japan

${ }^{42}$ Nagano National College of Technology, Nagano 381-8550, Japan

${ }^{43}$ Tokyo Metropolitan College of Industrial Technology, Tokyo 116-8523, Japan

${ }^{44}$ Institut d'Astrophysique de Paris, Université Pierre et Marie Curie, CNRS UMR7095, 98bis Boulevard Arago, 75014 Paris, France; beaulieu@iap.fr, marquett@iap.f

45 European Southern Observatory, Casilla 19001, Santiago 19, Chile; sbrillan@eso.org, dkubas@eso.org

${ }^{46}$ McDonald Observatory, 16120 St Hwy Spur 78 \#2, Fort Davis, TX 79734, USA; caldwell@astro.as.utexas.edu

${ }^{47}$ Department of Physics, Faculty of Natural and Agricultural Sciences, University of the Free State, P.O. Box 339, Bloemfontein 9300, South Africa; HoffmaMJ.SCI@mail.uovs.ac.za

${ }^{48}$ Lawrence Livermore National Laboratory, Institute of Geophysics and Planetary Physics, P.O. Box 808, Livermore, CA 94551-0808, USA; kcook@1lnl.gov

${ }^{49}$ CEA/Saclay, 91191 Gif-sur-Yvette Cedex, France; coutures@iap.fr

${ }^{50}$ Department of Physics, University of Rijeka, Omladinska 14, 51000 Rijeka, Croatia

51 Technische Universitaet Wien, Wieder Hauptst. 8-10, A-1040 Wienna, Austria; donatowicz@ tuwien.ac.at

52 LATT, Université de Toulouse, CNRS, France; pfouque@ ast.obs-mip.fr

${ }^{53}$ Instituto Nacional de Pesquisas Espaciais, Sao Jose dos Campos, SP, Brazil

${ }^{54}$ NASA Exoplanet Science Institute, Caltech, MS 100-22, 770 south Wilson Avenue, Pasadena, CA 91125, USA; skane@ipac.caltech.edu

${ }^{55}$ Perth Observatory, Walnut Road, Bickley, Perth 6076, WA, Australia; rmartin@physics.uwa.edu.au, andrew@ physics.uwa.edu.au

${ }^{56}$ South African Astronomical Observatory, P.O. Box 9, Observatory 7935, South Africa

${ }^{57}$ Space Telescope Science Institute, 3700 San Martin Drive, Baltimore, MD 21218, USA

${ }^{58}$ Niels Bohr Institutet, Københavns Universitet, Juliane Maries Vej 30, 2100 København Ø, Denmark

${ }^{59}$ Astronomisches Rechen-Institut, Zentrum für Astronomie der Universität Heidelberg (ZAH), Mönchhofstr. 12-14, 69120 Heidelberg, Germany

${ }^{60}$ Institute of Astronomy, University of Zielona Góra, Lubuska st. 2, 66-265 Zielona Góra, Poland

${ }^{61}$ School of Physics, University of Exeter, Stocker Road, Exeter EX4 4QL, UK

${ }^{62}$ Astrophysics Research Institute, Liverpool John Moores University, Liverpool CH41 1LD, UK

${ }^{63}$ European Southern Observatory, Karl-Schwarzschild-Straße 2, 85748 Garching bei München, Germany

${ }^{64}$ Deutsches SOFIA Institut, Universität Stuttgart, Pfaffenwaldring 31, 70569 Stuttgart, Germany

${ }^{65}$ SOFIA Science Center, NASA Ames Research Center, Mail Stop N211-3, Moffett Field CA 94035, USA

${ }^{66}$ Jodrell Bank Centre for Astrophysics, The University of Manchester, Oxford Road, Manchester M13 9PL, UK; Eamonn.Kerins@manchester.ac.uk

${ }^{67}$ Max Planck Institute for Solar System Research, Max-Planck-Str. 2, 37191 Katlenburg-Lindau, Germany

${ }^{68}$ Astronomy Unit, School of Mathematical Sciences, Queen Mary, University of London, Mile End Road, London, E1 4NS, UK

${ }^{69}$ Centre for Star and Planet Formation, Københavns Universitet, Øster Voldgade 5-7, 1350 København Ø, Denmark

${ }^{70}$ Departamento de Astronomía y Astrofísica, Pontificia Universidad Católica de Chile, Santiago, Chile

${ }^{71}$ Università degli Studi di Salerno, Dipartimento di Fisica “E. R. Caianiello," Via Ponte Don Melillo, 84085 Fisciano (SA), Italy

72 INFN, Gruppo Collegato di Salerno, Sezione di Napoli, Italy

${ }^{73}$ Istituto Internazionale per gli Alti Studi Scientifici (IIASS), Via G. Pellegrino 19, 84019 Vietri sul Mare (SA), Italy

${ }^{74}$ Armagh Observatory, College Hill, Armagh, BT61 9DG, UK

75 Institut für Astrophysik, Georg-August-Universität, Friedrich-Hund-Platz 1, 37077 Göttingen, Germany

${ }^{76}$ Dipartimento di Ingegneria, Università del Sannio, Corso Garibaldi 107, 82100 Benevento, Italy

${ }_{77}$ Bellatrix Astronomical Observatory, Via Madonna de Loco 47, 03023 Ceccano (FR), Italy

${ }^{78}$ Department of Physics, Sharif University of Technology, P.O. Box 11155-9161, Tehran, Iran

${ }^{79}$ Institut d'Astrophysique et de Géophysique, Allée du 6 Août 17, Sart Tilman, Bât. B5c, 4000 Liège, Belgium

${ }^{80}$ Astrophysics Group, Keele University, Staffordshire, ST5 5BG, UK

${ }^{81}$ INAF, Osservatorio Astronomico di Brera, 23806 Merate (LC), Italy

Received 2010 January 5; accepted 2010 July 8; published 2010 August 17

\footnotetext{
82 Sagan Fellow.

${ }^{83}$ RoboNet.

${ }^{84}$ Royal Society University Research Fellow.

${ }^{85}$ Einstein Fellow.
} 


\section{ABSTRACT}

We present the first measurement of the planet frequency beyond the "snow line," for the planet-to-star mass-ratio interval $-4.5<\log q<-2$, corresponding to the range of ice giants to gas giants. We find

$$
\frac{d^{2} N_{\mathrm{pl}}}{d \log q d \log s}=(0.36 \pm 0.15) \mathrm{dex}^{-2}
$$

at the mean mass ratio $q=5 \times 10^{-4}$ with no discernible deviation from a flat (Öpik's law) distribution in logprojected separation $s$. The determination is based on a sample of six planets detected from intensive follow-up observations of high-magnification $(A>200)$ microlensing events during 2005-2008. The sampled host stars have a typical mass $M_{\text {host }} \sim 0.5 M_{\odot}$, and detection is sensitive to planets over a range of planet-star-projected separations $\left(s_{\max }^{-1} R_{\mathrm{E}}, s_{\max } R_{\mathrm{E}}\right)$, where $R_{\mathrm{E}} \sim 3.5 \mathrm{AU}\left(M_{\text {host }} / M_{\odot}\right)^{1 / 2}$ is the Einstein radius and $s_{\max } \sim\left(q / 10^{-4.3}\right)^{1 / 3}$. This corresponds to deprojected separations roughly three times the "snow line." We show that the observations of these events have the properties of a "controlled experiment," which is what permits measurement of absolute planet frequency. High-magnification events are rare, but the survey-plus-follow-up high-magnification channel is very efficient: half of all high-mag events were successfully monitored and half of these yielded planet detections. The extremely high sensitivity of high-mag events leads to a policy of monitoring them as intensively as possible, independent of whether they show evidence of planets. This is what allows us to construct an unbiased sample. The planet frequency derived from microlensing is a factor 8 larger than the one derived from Doppler studies at factor $\sim 25$ smaller star-planet separations (i.e., periods 2-2000 days). However, this difference is basically consistent with the gradient derived from Doppler studies (when extrapolated well beyond the separations from which it is measured). This suggests a universal separation distribution across 2 dex in planet-star separation, 2 dex in mass ratio, and 0.3 dex in host mass. Finally, if all planetary systems were "analogs" of the solar system, our sample would have yielded 18.2 planets (11.4 "Jupiters," 6.4 "Saturns," 0.3 "Uranuses," 0.2 "Neptunes") including 6.1 systems with two or more planet detections. This compares to six planets including one twoplanet system in the actual sample, implying a first estimate of $1 / 6$ for the frequency of solar-like systems.

Key words: gravitational lensing: micro - planetary systems

Online-only material:

\section{INTRODUCTION}

To date, 10 microlensing-planet discoveries have been published, which permit, at least in principle, a measurement of planet parameter distribution functions. Of course, the size of this sample is small, both absolutely and relative to the dozens of planets that have been discovered from transit surveys and the hundreds from Doppler (radial velocity, hereafter RV) surveys. However, microlensing probes a substantially different part of parameter space from these other methods. The majority of RV planets, and the overwhelming majority of transiting planets are believed to have reached their present locations, generally well within the "snow line," by migrating by a factor $\gtrsim 10$ inward from their birthplace. By contrast, microlensing planets are generally found beyond the snow line, where gas giants (analogs of Jupiter and Saturn) and ice giants (analogs of Uranus and Neptune) are thought to form. Thus, it would be of substantial interest to compare the properties of microlensing planets, which have not suffered major migrations, to other planets that have.

In this paper, we analyze a sample of 13 high-magnification microlensing events that are well monitored by "follow-up" observation after their initial discovery. This represents a seemingly tiny fraction of the 5000+ microlensing events discovered to date. However, from the standpoint of planet detection, high-magnification events are incredibly efficient, accounting for about half of all microlensing-planet detections. Moreover, in contrast to their more common low-magnification cousins, high-magnification events are monitored essentially without reference to whether they have planets or not. This means that they constitute an unbiased sample from which one can measure the absolute frequency of planets simply by comparing the number of detected planets with the calculated detection efficiency of the event sample.

In Section 2, we document the historical developments that made high-mag microlensing planet searches feasible, both the intellectual recognition of the high sensitivity of well-monitored high-mag events and the practical advances that ultimately yielded a significant sample of such events to monitor.

In Section 3, we develop selection criteria that isolate an unbiased sample of high-mag events. We show that for a threshold magnification $A_{\max }>200$, the magnification distribution of well-monitored events is the same as that of the parent distribution from which they are selected. This statistical property is a reflection of our observational policy to activate intensive observations of high-magnification events and to continue observations until well past peak, regardless of whether planets are detected or not.

In Section 4, we derive an analytic expression for the sensitivity of an ensemble of high-magnification events monitored densely over their peak, and in Section 5, we calculate numerical sensitivities and use these to measure the frequency of ice giants and gas giants beyond the snow line. Finally, in Section 6, we discuss some implications of our results.

\section{HISTORY OF HIGH-MAGNIFICATION PLANET SEARCHES}

\subsection{Microlensing-Planet-Search Modes}

Microlensing planet searches were first suggested by Liebes (1964) and Mao \& Paczyński (1991). In their present incarnation, planet detection occurs through several different routes, with various degrees of "human intervention." At one extreme, 
planets can be detected simply on the basis of survey data, without even the knowledge that a planet was present until after the event is over. This is the plan for so-called second-generation microlensing planet surveys, which will permit rigorous, particlephysics-like objective analysis of a "controlled experiment." In fact, Tsapras et al. (2003) and Snodgrass et al. (2004) have already carried out such analyses of first-generation survey-only data. However, to date only one secure microlensing planet has been discovered from survey-only data, MOA-2007-BLG192Lb (Bennett et al. 2008).

Rather, most microlensing planet detections have taken place through a complex interplay of survey and follow-up observations. Only a year after the first microlensing events were discovered, the MACHO collaboration issued an IAU circular urging follow-up observations of a microlensing event, and the OGLE collaboration initiated its Early Warning System (EWS; Udalski et al. 1994; Udalski 2003), which regularly issued "alerts" of ongoing microlensing events, usually well before peak, as soon as they were reliably detected. EWS, together with a similar program soon initiated by the MACHO collaboration (Alcock et al. 1996) with their much larger camera, enabled the formation in 1995 of the first microlensing follow-up teams, PLANET (Albrow et al. 1998) and MACHO/GMAN (Alcock et al. 1997), and soon thereafter, MPS (Rhie et al. 1999). The EROS team issued only a few alerts, but one of these led to the first mass measurement of an unseen object (An et al. 2002), and the first spatially resolved high-resolution spectrum of another star (Castro et al. 2001). OGLE-II inaugurated wide-field observations as well in 1998. In order to cover large areas of the sky (even with wide-field cameras), the survey teams would generally obtain only $\sim 1$ point per night per field. Since typical planetary deviations from "normal" (point-lens) microlensing light curves last only of order a day or less, this was not generally adequate to detect a planet. Hence, Gould \& Loeb (1992) already advocated the formation of follow-up teams that would choose several favorable events to monitor more frequently, using telescopes on several continents to permit $24 \mathrm{hr}$ coverage. In 2000, MACHO ceased operations, but a new survey group, MOA, had already begun survey observations.

Once this synergy between survey and follow-up teams was established, it evolved quickly on both sides. Both types of teams developed the capacity for "internal alerts," whereby real-time photometry was quickly analyzed to find hints of an anomaly. If these hints were regarded as sufficiently interesting, they would trigger additional observations by the team. The first such alert was by MACHO/GMAN in 1996 and several followed the next year from PLANET. In 2003, OGLE developed the Early EWS, which automatically alerted the observer to possible anomalies, who then would make additional observations and, if these were confirming, publicize them to the community. OGLE also pioneered making their data publicly available, which greatly facilitated follow-up work. These developments generally evolved into a system of mutual alerts, open transfer of data between teams, and active ongoing email discussion of developing events.

The first secure microlensing planet, OGLE-2003-BLG235Lb (Bond et al. 2004) was discovered by means of such an internal alert. The MOA team noticed deviations in their data (initially not definitively interpreted), and initiated intensive follow-up of this event. In retrospect, one can see that even without this internal alert, the combined OGLE and MOA data would have been sufficient to show that the star had a companion. However, the normal survey data would not have permitted one to tell whether this companion was a planet or a low-mass star (or possibly a brown dwarf).

Detections of this type are at the opposite extreme from the pure-survey detection, which can be modeled as a controlled experiment. Without understanding the efficiency at which the observers issue their real-time alerts, one cannot measure the absolute rate of planets from observations that are triggered by the presence of the planet itself. Another planet, OGLE-2007BLG-368Lb (Sumi et al. 2010) falls partly into this category. In this case, the alert was triggered by ARTEMIS (Dominik et al. 2008), whose ultimate goal is to communicate such alerts directly to the follow-up telescopes without human interference (and so bring such alerts into the fold of "rigorous controlled experiments"), although at this stage ARTEMIS alerts were still vetted by humans. A further anomaly was soon detected by the MOA observer. But even without follow-up observations triggered by these alerts, this companion probably could have been constrained to be planetary, although with larger errors, so this detection could still be integrated into the "controlled experiment" framework even without trying to model the alert process.

However, if follow-up observations are carried out without being significantly influenced by the possible presence of a planet, then these also can be treated as a controlled experiment, and absolute rates can be derived using either the method of Gaudi \& Sackett (2000) or of Rhie et al. (2000). Such an analysis was carried out for the first 43 events monitored by the PLANET Collaboration (Albrow et al. 2001; Gaudi et al. 2002). In particular, since no planets were detected (and so only upper limits obtained), there was no possibility of recognition of a possible planet playing any role in the observation strategy. But, as emphasized above, once planets started to be discovered, the situation became more nuanced.

One path toward obtaining follow-up observations that are triggered on potentially planetary anomalies, without compromising the "controlled experiment" ideal, is to establish robotic triggering programs that communicate directly to robotic telescopes. This has been the goal of the RoboNet Collaboration since its inception in 2004 (Tsapras et al. 2009). Algorithms for detecting anomalies in robotically acquired real-time data (Dominik et al. 2007, 2008) and directing robotic telescopes (Horne et al. 2009) have been devised and are being further developed, and will expand their scope as the Las Cumbres Observatory Global Telescope Network (LCOGT) itself expands. But to date, the most effective RoboNET observations have been "hand triggered" (e.g., for OGLE-2007-BLG-349Lb).

In spite of this nuanced situation, Sumi et al. (2010) were nevertheless able to extract relative frequencies of planets from the ensemble of all 10 microlensing planets. They argued that each of these planet detections was characterized by a sensitivity $g(q) \propto q^{m}$, where $q$ is the planet/star mass ratio. While $m$ varies from event to event, they argued that $m=0.6 \pm 0.1$ was an appropriate average value. They then fit the ensemble of detections to a power-law mass distribution $f(q) d \log q \propto$ $q^{n} d \log q$ and found $n=-0.68 \pm 0.20$. But they did not attempt to extract absolute frequencies from their sample.

\subsection{The High-magnification Technique}

Here, we analyze an important subclass of microlensing planet searches: high-magnification events that are intensively monitored over the peak. The observations of these events are always frenetic, sometimes even comical, so it may seem surprising that they nevertheless constitute a "controlled experiment," or very nearly so, and hence are subject to rigorous analysis. 
More than a decade ago, Griest \& Safizadeh (1998) pointed out that high-mag events are much more sensitive to planets than their far more common cousins, the low-magnification events. The reason is fairly simple. Magnification is, almost by definition, the inverse second derivative of the time-delay surface. Hence, high-magnification implies small second derivatives and, consequently, extreme instability of the images. During the peak of a high-mag point-lens event, the two images sweep around opposite sides of the Einstein ring, together probing almost $2 \pi$. If they encounter a small planet near the Einstein ring or a large one farther away, these highly unstable images will be easily perturbed leading to a pronounced deviation in magnification. The key point is that the planet can be at a broad range of separations and at virtually any angle relative to the source trajectory and still create a caustic that will perturb the light curve.

However, following this seminal paper, microlensing followup monitoring groups continued to focus primarily on garden variety microlensing events, which are only sensitive to socalled "planetary caustics". These are formed by the action of the primary-star gravitational field on the planet gravitational field, and as such are bigger and so have larger cross sections than central caustics. At first sight, this makes them more favorable targets, but this conclusion only holds if one has unlimited telescope time for monitoring. Then one would monitor all available events (and hence primarily low-mag events) and would find most planets in these events (just because the caustics have a larger cross section). But if observing resources are limited, then one should focus these on high-mag events because these can be predicted (at least in principle) from the pre-peak part of the light curve and have individually higher sensitivity to planets. By contrast, there is no way to predict that a source is approaching a planetary caustic.

Nevertheless, within the context of continued focus on planetary caustics in normal events, there were significant efforts to take advantage of high-mag events as well. In 1998 MPS issued the first high-mag alert for MACHO-98-BLG-35, which received an enthusiastic response from the MOA group, leading to the possible detection of a planet (Rhie et al. 2000; Bond et al. 2002b), but with too-low significance to be confident. From the inception of its survey, MOA made real-time alerts of high-magnification events a priority and attempted to organize follow-up from other continents, with 10 such alerts the first year (Bond et al. 2002a). The most spectacular success of this program was MOA-2003-BLG-32/OGLE-2003-BLG-219, which was densely sampled over its $A_{\max }=520$ peak by the Wise observatory in Israel after such an alert, and which yielded the best upper limits on planetary companions to a lens to that date (Abe et al. 2004). Theoretical work was also done to optimize observations of high-mag events for planet sensitivity (Rattenbury et al. 2002).

\subsection{Evolution of $\mu F U N$ Strategy}

When the Microlensing Follow Up Network ( $\mu$ FUN) began operations in 2001, it followed the already-established model of follow-up observations, which did include high-mag events (Yoo et al. 2003), but did not emphasize them. However, three things happened to change its orientation toward concentrating on high-mag events. First, the OGLE-III survey came on line in 2002 with a discovery rate of 350 events per year, moving up to 600 events per year in 2004. This compared with 40-80 events per year discovered by OGLE-II in 1998-2000. The number of events alerted per year has a direct impact on whether or not one is in the regime of "limited" or "unlimited" resources. Before 2002, if one restricted oneself to high-mag events, one would mostly be sitting on one's hands. For example, when Albrow et al. (2001) and Gaudi et al. (2002) analyzed five years of PLANET Collaboration data, they reported only two events with $A_{\max }>100$. OGLE-III dramatically changed that situation. More recently, the MOA collaboration inaugurated MOA-II (2007 first full season), which has had the net effect of increasing the total rate of reported events by about $50 \%$.

Second, $\mu$ FUN began attracting the intrinsically "limited" observing resources of amateur astronomers. These contrast with the larger dedicated professional observatories in two key ways. First, the observers generally cannot observe all night, every night, or they will be unable to keep their day jobs. Second, the smaller apertures of their telescopes restrict them to relatively brighter targets. Both "limitations" naturally drive amateurs to high-mag events, which have a bigger chance of science payoff and are brighter (because highly magnified). Moreover, there is one crucial dimension in which amateurs are not limited: they have completely free and almost instantaneous access to their telescopes at any time. Thus, while dedicated follow-up telescopes are typically operated only in the 3-4 month core of the season (when microlensing targets are observable for at least half the night), amateurs can react to alerts deep into the wings of the season, close to doubling the number of high-mag events that can be monitored. In 2004, one amateur began observing $\mu \mathrm{FUN}$ targets on her own initiative. She requested regular alerts and began organizing other amateurs to join in, who in turn self-organized a network. About half of the $\mu \mathrm{FUN}$ authors of this paper are amateurs.

Third, $\mu \mathrm{FUN}$ had to become aware of this changed situation, i.e., that it had moved from the domain of unlimited to limited resources. This transition was partly aided by the fact that $\mu \mathrm{FUN}$ access to two of its professional telescopes (Wise and SMARTS CTIO) was limited by their being shared resources. But throughout 2003-2004, $\mu$ FUN "straddled two horses," focusing on high-mag events when available, but trying to keep to the old planetary-caustic strategy most of the time. Preparations for the 2005 season were significantly influenced by preliminary work (ultimately, Dong et al. 2006) showing that unless high-mag events were intensively monitored over their peak, much of their sensitivity to planets is compromised.

Then in 2005 April, $\mu \mathrm{FUN}$ intensively followed the (by those days' standards) high-mag event OGLE-2005-BLG-071, which resulted in the detection of the second microlensing planet (Udalski et al. 2005). This detection led to $\mu \mathrm{FUN}$ consciously changing its orientation, procedures, recruitment, etc., with the aim of focusing primarily on high-mag events.

\subsection{A Controlled Experiment}

From 2005 onward, considerable effort has gone into identifying potential high-mag events, and in some cases obtaining additional data to improve the prediction of $A_{\max }$. If an event is deemed a plausible high-mag candidate, then observers are notified by email, without necessarily being urged to observe, just to put them on alert. Once high magnification seems probable, observations are requested with various degrees of urgency. The urgency is conditioned by the fact that peak sensitivity is usually less than a day (the normal human cycle time) but more than a few hours (so requiring observations from multiple continents). Many factors enter into the quality of the final light curve, including weather conditions on six continents plus Pacific Islands, observer availability, communication problems, 
etc. Indeed, it is difficult to convey the level of chaos during one of these events.

Despite (and also because of) this chaos, the resulting data stream generally retains the character of a "controlled experiment." In the "ideal incarnation" of this search mode, the event is recognized (with greater or lesser certainty) to be approaching a high-magnification peak, and an alert is issued to interested observers urging them to observe it intensively over the predicted peak. The observations take place regardless of whether the planet is present or not. The very chaos, remoteness of observing locations, and communication problems make it difficult to gain knowledge of planetary perturbations until after the key observations are over.

\section{SELECTION CRITERIA AND DATA}

We begin by designing criteria for selecting events and planets to be included in the analysis that enable the detections and nondetections to be analyzed on the same footing. This is essential to the goal of defining a data sample that can be treated as a "controlled experiment."

\subsection{Selection Criteria for Events}

(E1) High-cadence ( $<10$ minutes) $\mu \mathrm{FUN}$ data over some portion of the peak.

(E2) High-cadence data covering at least one wing of the peak, $\left|t-t_{0}\right|<t_{\text {eff }}$, with no major gaps.

(E3) High signal-to-noise ratio $(\mathrm{S} / \mathrm{N})$ data $\left(\sum_{i} \sigma_{i}^{-2}>\right.$ $50,000)$ covering the other wing, where the $\sigma_{i}$ are the data-point errors in magnitudes.

(E4) Light curve is not dominated by binary (non-planetary) features.

Here, $t$ is time and $t_{0}$ is the time when $u=u_{0}$, i.e., the time of the closest lens-source approach. We define $t_{\text {eff }} \equiv u_{0} t_{\mathrm{E}}$ for point-lens/point-source events, where $t_{\mathrm{E}}$ is the Einstein crossing time. For point-lens events that suffer finite-source effects, we generalize this definition to the time interval during which the magnification is within $\sqrt{2}$ of the peak. And for planetary events, we further generalize it to the time interval that the magnification would have been within $\sqrt{2}$ of the peak if the lensing star had lacked planets.

We now justify these criteria.

In addition to reflecting the fact that we are summarizing $\mu$ FUN work, criterion (E1) ensures that we can rigorously review the available data on $\sim 3000$ events discovered during 2005-2008, and reduce them to a manageable subset of "only" 315 that can be investigated using $\mu$ FUN files. These 315 were then quickly pared down to a few dozen events that are consistent with high-magnification and actually meet criterion (E1).

Criteria (E2) and (E3) should be considered together. Our underlying requirement is to have enough coverage of the event so that planetary deviations that give rise to a $\Delta \chi^{2}=500$ deviation (for the rereduced data set), have a high probability of yielding a unique scientific interpretation. See criterion (P2), below. These two criteria are basically derived from the experience analyzing the event OGLE-2005-BLG-169 (Gould et al. 2006), which does somewhat better than barely satisfy both. The analysis of this event was already fairly difficult because of the multiple $\chi^{2}$ minima and would have been quite degenerate if, for example, it lacked high $\mathrm{S} / \mathrm{N}$ data on the rising side. Hence, it would not have led to a publishable planet with a reasonably well-defined mass ratio.
Table 1

Monitored Events with Magnification $A>100$

\begin{tabular}{lccccc}
\hline \hline Name & $A_{\max }$ & $t_{0}(\mathrm{HJD})$ & $t_{\mathrm{E}}$ & $M / M_{\odot}$ & Method \\
\hline OGLE-2007-BLG-224 & 2424 & 4233.7 & 7 & $0.056 \pm 0.004$ & $M=\theta_{\mathrm{E}} / \kappa \pi_{\mathrm{E}}$ \\
\hline OGLE-2008-BLG-279 & 1600 & 4617.3 & 101 & $0.64 \pm 0.10$ & $M=\theta_{\mathrm{E}} / \kappa \pi_{\mathrm{E}}$ \\
\hline OGLE-2005-BLG-169 & 800 & 3491.9 & 43 & $0.49_{-0.29}^{+0.23}$ & $\mathrm{GM} \oplus \theta_{\mathrm{E}} \oplus t_{\mathrm{E}}$ \\
\hline MOA-2007-BLG-400 & 628 & 4354.6 & 14 & $0.30_{-0.12}^{+0.19}$ & $\mathrm{GM} \oplus \theta_{\mathrm{E}} \oplus t_{\mathrm{E}}$ \\
\hline OGLE-2007-BLG-349 & 525 & 4348.6 & 121 & $\sim 0.6$ & $M=\theta_{\mathrm{E}} / \kappa \pi_{\mathrm{E}}$ \\
\hline OGLE-2007-BLG-050 & 432 & 4222.0 & 68 & $0.50 \pm 0.14$ & $M=\theta_{\mathrm{E}} / \kappa \pi_{\mathrm{E}}$ \\
\hline MOA-2008-BLG-310 & 400 & 4656.4 & 11 & $\leqslant 0.67 \pm 0.14$ & $\mathrm{AO}$ \\
\hline OGLE-2006-BLG-109 & 289 & 3831.0 & 127 & $0.51_{-0.04}^{+0.05}$ & $M=\theta_{\mathrm{E}} / \kappa \pi_{\mathrm{E}}, \mathrm{AO}$ \\
\hline OGLE-2005-BLG-188 & 283 & 3500.5 & 14 & $0.16_{-0.08}^{+0.21}$ & $\mathrm{GM} \oplus \theta_{\mathrm{E}} \oplus t_{\mathrm{E}}$ \\
\hline MOA-2008-BLG-311 & 279 & 4655.4 & 18 & $0.20_{-0.09}^{+0.26}$ & $\mathrm{GM} \oplus \theta_{\mathrm{E}} \oplus t_{\mathrm{E}}$ \\
\hline MOA-2008-BLG-105 & 267 & 4565.8 & 10 & & \\
\hline OGLE-2006-BLG-245 & 217 & 3885.1 & 59 & & \\
\hline OGLE-2006-BLG-265 & 211 & 3893.2 & 26 & & \\
\hline
\end{tabular}

OGLE-2007-BLG-423 $157 \quad 4320.3 \quad 29$

OGLE-2005-BLG-417 $108 \quad 3568.1 \quad 23$

Criterion (E4) is adopted for two reasons. First, in contrast to point-lens events, most binary events are not modeled with sufficient precision to measure the $u_{0}$ parameter well enough to construct a well-defined sample of events with maximum magnification greater than some threshold $A_{\max }$. Second, the problem of detecting planets in the presence of light-curve features dominated by a binary is not well understood. Hence, the results we derive here really apply to stars not giving rise to strong binary features, which excludes roughly 3\%-6\% of all stars (Alcock et al. 2000; Jaroszyński et al. 2006).

Table 1 lists all events from 2005 to 2008 that satisfy these four criteria and that had peak magnifications $A_{\max }>100$. Column 1 is the event name, Column 2 is the maximum magnification, Column 3 is the time of closest approach between the source and lens $t_{0}$, Column 4 is the Einstein timescale $t_{\mathrm{E}}$, Column 5 gives the mass of the lens star for cases that it is known, and Column 6 gives the method by which it is derived. These lens masses and methods are discussed in Section 5.2. The events are listed in inverse order of $A_{\max }$.

Figure 1 shows the cumulative distribution $A_{\max }^{-1}$ for the 15 events in Table 1. It displays a clear break at $A_{\max }=200$. Below this value, the distribution is uniform in $A_{\max }^{-1}$, which is the expected behavior for a complete sample (ignoring finite-source effects), i.e., uniform in $u_{0}$, which is the impact parameter in units of the Einstein radius. The dashed line, with slope of $d N_{\mathrm{ev}} / d A_{\max }^{-1}=2600$ is a good match to the $A_{\max }>200$ data. Cohen et al. (2010) found such uniformity for the underlying sample of OGLE events in 2008, with a slope of $d N_{\mathrm{ev}} / d u_{0}=1080$ for $u_{0}<0.05$. From this comparison, we learn two things. First, $\mu \mathrm{FUN}$ was aggressive enough to achieve a uniform subsample only for events with $A_{\max }>200$. Second, $\mu$ FUN was able to intensively monitor half of all events in the $A_{\max }>200$ subsample. That is, assuming that OGLE found similar numbers of high-mag events in 2005-2007, and accounting for the fact that MOA found $50 \%$ more events (not found by OGLE) in 2007-2008, the full sample of high-mag events was about $d N_{\mathrm{ev}} / d u_{0} \sim 5 \times 1080=5400$, of which $\mu$ FUN effectively monitored about $48 \%$. 


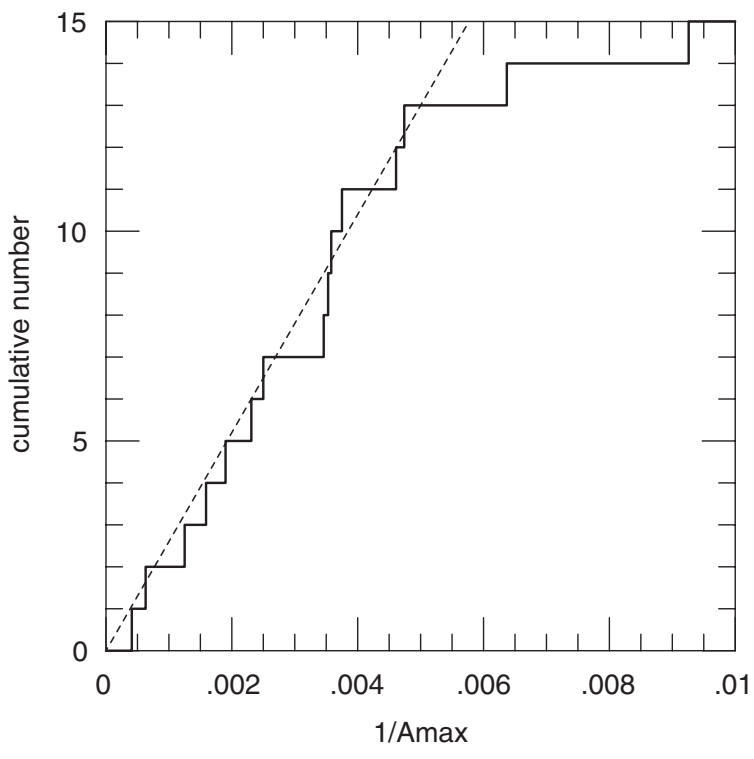

Figure 1. Cumulative distribution of inverse maximum magnification $A_{\max }^{-1}$ for high-mag events observed by $\mu$ FUN during the years 2005-2008. The distribution is uniform in $A_{\max }^{-1}$ for high-mag events $A_{\max }>200$, with a slope of $d N_{\mathrm{ev}} / d A_{\max }^{-1}=2600$ (dashed line). A "controlled experiment" therefore requires a selection criterion $A_{\max }>200$ (see Section 3.1).

Although it may not be immediately obvious, Figure 1 implies that we must impose a fifth criterion.

(E5) $A_{\max }>200$.

Figure 1 demonstrates that $\mu \mathrm{FUN}$ was substantially less enthusiastic about events $A_{\max }<200$ than $A_{\max }>200$, whether because it simply did not act on events known in advance to be in the former category or just became less enthusiastic about observations once these events were recognized near peak not to be extremely magnified. This bias is a natural consequence of $\mu$ FUN's limited observing resources (as discussed in Section 2): there are four times as many events with $A_{\max }>50$ as $A_{\max }>200$ and their duration of peak, $2 t_{\mathrm{eff}} \sim 2 t_{\mathrm{E}} A_{\max }^{-1}$ lasts four times as long, so 16 times more observing resources would be required to follow them all. Hence, if an event proved midway to be one that the objective evidence demonstrates $\mu \mathrm{FUN}$ cared less about, there would be a tendency on the part of observers to slacken efforts (whether or not the internal alert was officially called off). Then the event would have less chance of meeting the selection criteria. But if a planet were detected during the peak observations (and there is a greater chance of recognizing a planet in real time for lower $A_{\max }$ because the peak lasts longer) then observations would not slacken, but rather intensify. Since this bias cannot be rigorously quantified, planets and nondetections from $A_{\max }<200$ events must both be excluded from the sample.

\subsection{Selection Criteria for Planets}

(P1) Planet must be discovered in an event that satisfies (E1)-(E5).

(P2) Planetary fit yields improvement $\Delta \chi^{2}>500$.

(P3) Planet-star mass ratio $q$ must lie in the range

$$
q_{-}<q<q_{+}, \quad q_{-}=10^{-4.5}, \quad q_{+}=10^{-2} .
$$

Criterion (P1) is self-evident but is stated explicitly for completeness and emphasis. Criterion (P2) may appear at first sight somewhat draconian, but it is realistic. To explain this,
Table 2

Planets in Densely Monitored High-mag Events

\begin{tabular}{lccccc}
\hline \hline Name & $\log u_{0}$ & $\log \rho$ & $\log q$ & $|\log s|$ & $\left|\log s_{\max }\right|$ \\
\hline OGLE-2005-BLG-169Lb & -2.9 & -3.4 & -4.1 & 0.009 & 0.19 \\
\hline OGLE-2006-BLG-109Lb & -2.5 & -3.5 & -2.9 & 0.20 & 0.39 \\
\hline OGLE-2006-BLG-109Lc & -2.5 & -3.5 & -3.3 & 0.017 & 0.27 \\
\hline OGLE-2007-BLG-349Lb & -2.7 & -3.3 & -3.5 & 0.099 & 0.48 \\
\hline MOA-2007-BLG-400Lb & -3.6 & -2.5 & -2.6 & 0.47 & 0.55 \\
\hline MOA-2008-BLG-310Lb & -2.5 & -2.3 & -3.5 & 0.035 & 0.14
\end{tabular}

we first note that among the six planets listed in Table 2, the "weakest" detection is $\Delta \chi^{2}=880$, which is for MOA-2008BLG-310Lb (Janczak et al. 2010). Now, it is certainly possible to recognize systematic residuals from a point-lens fits "by eye" at a much lower level, even $\Delta \chi^{2}=100$. Indeed, Batista et al. (2009) argued that no systematic residuals were present in the fit to OGLE-2007-BLG-050 at a much lower level, $\Delta \chi^{2}=60$. But if such deviations had been observed in an event, this would not have necessarily enabled discovery of a planet, where "discovery" here means "publication." First, $\Delta \chi^{2}=100$ in the final, rereduced and carefully cleaned data implies something like $\Delta \chi^{2}=50$ in the standard pipeline data, and systematic deviations due to a planet at this level would probably not be recognized as significant, i.e., clearly distinguishable from systematics that appear in dozens of other events, and which just reflect instrumental, weather, or data-reduction problems. But more to the point, even if the unrereduced-data were $\Delta \chi^{2}=100$, triggering strenuous efforts to clean and rereduce the data set, resulting in, say, a $\Delta \chi^{2}=200$ improvement, it is far from clear that this deviation (even if strongly believed to be real) would lead to a publishable planet detection. This is because, in addition to obtaining an acceptable fit to the data, such a paper would have to demonstrate that there could be no acceptable fits to the data for non-planetary solutions. We have already designed criteria (E2) and (E3) to eliminate those events for which very high $\Delta \chi^{2}$ is possible without leading to a unique interpretation (due to incomplete coverage of the deviation). But we still must set the threshold high enough so that if an anomalous event survives criteria (E2), (E3), and (P2), it has a small chance of being ambiguous in its interpretation. Our best estimate of this, from experience fitting events, is $\Delta \chi^{2}=500$. However, we regard other values in the range $350-700$ as also being plausible candidates for this threshold. We will show in Section 5 that our basic conclusions are robust to changes within this range.

The upper boundary in Equation (1), criterion (P3), is necessary because at high mass ratios $q$, one cannot be confident that the event will not be rejected (consciously or unconsciously) as a "brown dwarf" or "low-mass star," and therefore not be monitored as intensively as it might be (and so not pass criteria (E2) and (E3)). To illustrate this, we review how OGLE-2008-BLG513Lb (J. C. Yee et al., in preparation) was "almost rejected" as a binary, even though it is probably a planet. This event has a large, strong, resonant caustic that was initially mistaken for a binary. During the long intra-caustic period, it was realized that the companion might be a planet, and that intensive observations of the caustic exit would be necessary to resolve this question. Such observations were obtained, and from these we know that the impact parameter was $u_{0} \sim 0.039$, so $A_{\max } \sim 26$, which means that the event fails criterion (E5) and so is excluded from our sample. But if these data had not been obtained, then $u_{0}$ 
r - projected planet/star separation (AU)

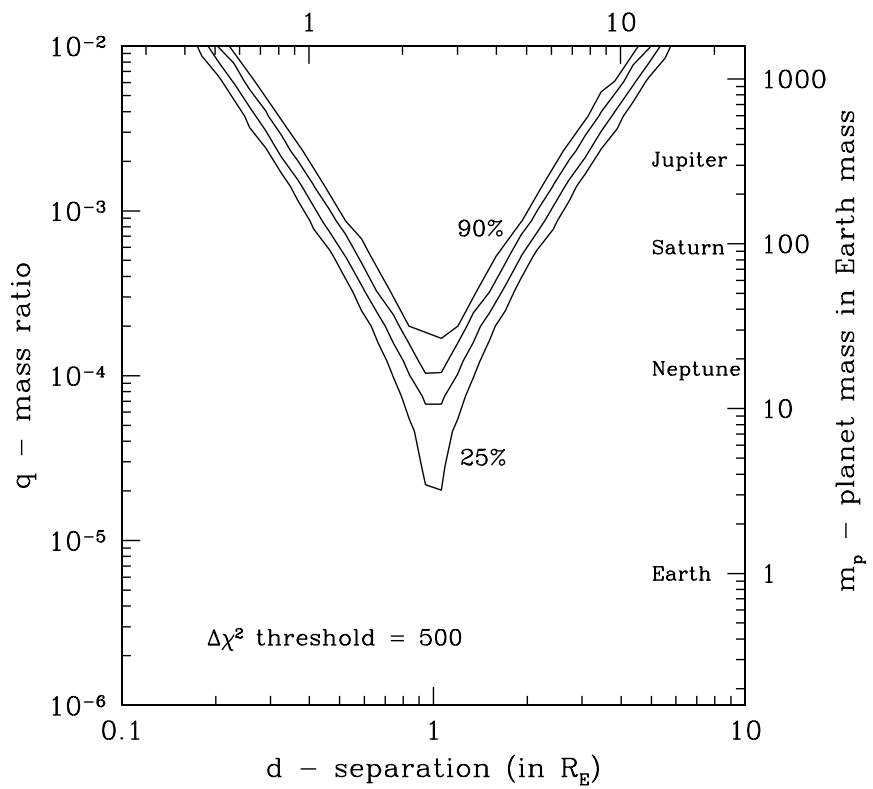

Figure 2. Triangle diagram for OGLE-2007-BLG-050 (adapted from Figure 9 of Batista et al. 2009) showing sensitivity to planets of planet/star mass ratio $q$ and planet-star-projected separation $d$ (alias $s$ in the current paper). Planets within the contours would be detectable at $\Delta \chi^{2}>500$ for $25 \%, 50 \%, 75 \%$, and $90 \%$ of source-lens trajectories. Since these trajectories are random, the contours reflect the probability of detecting the planet at a given $s$ and $q$. The slope of the contours is $\eta=0.32$.

for this event would not be known, and it would not be known whether the event was in the sample or not, and if it were, whether the companion was a planet or not.

Why does this example then not just prove that the whole concept of "controlled experiment" is unviable? The answer is given by Figures 2 and 3. One sees from these that at high mass ratios, $q \sim 10^{-2}$, there is an extremely wide range of $s$ for which the planet is "detectable." Only a small fraction of these "detectable" events have $s \sim 1$, which produce large resonant caustics that might be mistaken for binaries. Hence, while in principle some of these "detections" might be lost to this confusion, the great majority would not cause any confusion. The reason that planets like OB08513Lb make their way into the detections at all, despite their relative rarity, is that the caustics are so large that they are detectable over a wide range of $u_{0}$, only a small fraction of which would pass the "high-mag" criteria of Section 3.1. Nevertheless, as $q$ grows, this potential problem grows with it. We adopt $\log q_{+}=-2$, but recognize that values ranging from -2.3 to -1.8 might also have been plausible choices. (Note that OGLE-2008-BLG-513 itself has a mass ratio $q \sim 0.026$, well above our adopted $q_{+}$.)

The lower boundary $q_{-}=10^{-4.5}$ is established because of concerns of the real "detectability" of low-mass planets in the presence of higher-mass planets in the same system. In the method of Rhie et al. (2000), which we employ in Section 5.1, planet sensitivity is determined by fitting simulated star-planet light curves that are constructed to have the same error properties as the actual data, to point-lens models. If the best such model increases $\chi^{2}$ by more than a given threshold (say $\Delta \chi^{2}>500$ ), then the planet is said to be detectable. Since this method directly mimics the process of planet detection for single-planet systems, it is a good way to characterize the detectability of such systems. But high-magnification events are particularly sensitive to multiple planets (Gaudi et al. 1998) and why should

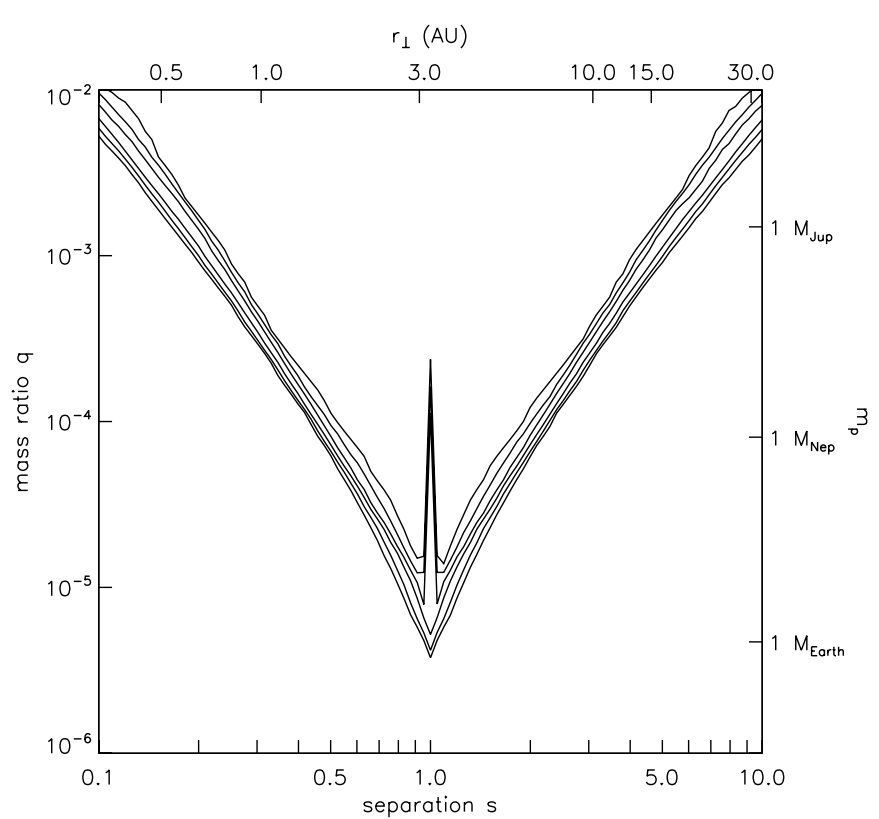

Figure 3. Triangle diagram of planet sensitivities for OGLE-2008-BLG-279 (adapted from Figure 7 of Yee et al. 2009). Similar to Figure 2 except with contours of $\Delta \chi^{2}>500$ detectability with $10 \%, 25 \%, 50 \%, 75 \% 90 \%$, and $99 \%$ probability. Contour slope is $\eta=0.35$.

this approach tell us anything about the detectability of a second planet in a system already containing one planet? As first shown by Bozza et al. (1999), the net perturbations of such two-planet high-magnification light curves usually "factor" into the sum of perturbations induced by each planet separately. For example, the only published two-planet system has this property (Gaudi et al. 2008; Bennett et al. 2010). Of course, the factoring is not perfect, but in this real case (and in many simulated cases), once the dominant-planet perturbation is removed, the secondary perturbation is easily recognized, leading to an excellent starting point for a combined fit to both planets simultaneously. For reasonably comparable planet mass ratios, the only exception to this is if the planet-star axes are closely (within $\lesssim 20^{\circ}$ ) aligned (Rattenbury et al. 2002; Han 2005). In this case, the singleplanet fit still fails, but the residuals to this fit are not easily recognizable. While such difficulties might impede recognition of the second planet, the required alignment is so close, that such cases would be a small minority of two-planet systems.

However, this factoring has only been studied in detail for planets with relatively comparable masses (Han 2005). The situation may not be as simple when the mass ratio of the two planets is extreme. Based on analysis of the events listed in Table 2, below, we cannot be confident of excluding all "second planets" with $q<10^{-5}$. To be "conservative," we have moved the boundary to $q_{-}=10^{-4.5}$.

Because both $q_{-}$and $q_{+}$have some uncertainty, we must ask how robust our conclusions are to changes in these parameters within a reasonable range. We show in Section 5 that our basic conclusions about planet frequency are not seriously affected by uncertainty in $q_{-}$and $q_{+}$. However, these uncertainties will prevent us from deriving a slope of the mass-ratio function.

Six planets satisfy criteria (P1)-(P3). Table 2 displays their characteristics. Columns $2-5$ are the parameters $\left(u_{0}, \rho, q,|\log s|\right)$ measured from the event, where $\rho$ is the source radius in units of the angular Einstein radius $\theta_{\mathrm{E}}$. In several cases, there is an unresolved $s \leftrightarrow s^{-1}$ degeneracy, which 
is irrelevant to the current study, so we just display the absolute value of the log. The final column is the maximum detectable value of $|\log s|$ according to calculations reported in Section 5.1.

\subsection{Were Discovery Observation Cadences Really Independent of the Planet?}

Were the observations that led to the discovery of the six planets in fact carried out independent of the presence of the planet? The key point is that it is our policy to intensively monitor high-mag events, whether or not they have planets, and this policy is reflected in the data streams of the planetbearing events. For three of these events, OGLE-2005-BLG169Lb (Gould et al. 2006), MOA-2007-BLG-400Lb (Dong et al. 2009b), and MOA-2008-BLG-310Lb (Janczak et al. 2010), the planet was not recognized until after the event had returned to baseline. All three were monitored very intensively from the time they were alerted as high-mag until well past peak. (OGLE2005-BLG-169 was not alerted until near peak due to poor weather and technical problems.) OGLE-2007-BLG-349 (S. Dong et al., in preparation) was recognized to have a significant deviation possibly due to a planet based on observations in Chile, $36 \mathrm{hr}$ after the call for intensive observations based on its highmag trajectory, and roughly $7 \mathrm{hr}$ after observations had begun in South Africa. While it is true that reports of this potential planet heightened excitement, and could in principle have increased the commitment of observers to get observations, the data record shows that the density of observations (from four continents plus Oceania) did not qualitatively change after the potentially planetary anomaly was recognized. This is the only one of the six planets in our sample that is not yet published. The reason is that the system contains a third body, which has proven difficult to fully characterize. However, the characteristics of OGLE2007-BLG-349Lb are very well established, and the third body is certainly not in the mass range being probed in the current analysis. Hence, we feel confident including this planet in the sample.

The two planets OGLE-2006-BLG-109Lb,c (Gaudi et al. 2008; Bennett et al. 2010) require closer examination. OGLE2006-BLG-109 was recognized to be an interesting event almost 10 days prior to peak due to detection of what turned out to be the resonant caustic of OGLE-2006-BLG-109Lc, the Saturn massratio planet in this system. This anomaly did indeed trigger some additional observations, which did help characterize this planet. But a review of email communications that initiated followup observations during the event shows that far more intensive observations were triggered several days later, after the event had appeared to return to "normal" (point-lens-like) microlensing, exactly by its high-mag trajectory. Although these emails remark on the possible presence of a planet, they place primary emphasis on this being an otherwise "normal" microlensing event that was reaching extreme magnification. (Note that the appearance of extreme magnification was not itself an artifact of the presence of planets(s), but was simply due to low source-lens impact parameter.) It was the intensive observations from New Zealand, triggered by these emails, that captured the "central structure" of the caustic due to OGLE-2006-BLG-109c. These would have enabled basic characterization of this planet even without the flurry of follow-up observations 10 days earlier. Moreover, it was the same email that triggered intensive observations from two widely separated locations (Israel and Chile), that enabled detection of the "central caustic" due to OGLE-2006-BLG$109 \mathrm{Lb}$, the Jupiter mass-ratio planet. Thus, all detections were in reasonable accord with the "controlled experiment" ideal.

\section{ANALYTIC TREATMENT}

\subsection{Triangle Diagrams}

Batista et al. (2009) and Yee et al. (2009) recently analyzed the sensitivity to planets of two events in our sample from Table 1, OGLE-2007-BLG-050 and OGLE-2008-BLG-279, respectively. Figures 2 and 3 are versions of their results, but with the detection threshold used in this paper, $\Delta \chi^{2}=500$. In contrast to the wide range of detection-sensitivity morphologies shown in Figure 8 of Gaudi et al. (2002), both of these diagrams have a simple triangular appearance, which is basically described by a two-parameter equation

$$
|\log s|_{\max }(q)=\eta \log \frac{q}{q_{\min }},
$$

where $q$ is the planet/star mass ratio, $s$ is the planet-star projected separation in units of the Einstein radius, $\eta$ is the slope of the triangle, and $q_{\min }$ defines the "bottom" of the triangle. Planets lying inside the triangle are detected with $100 \%$ efficiency and those lying outside are undetectable. The boundary region is quite narrow. In principle, planet detection is a function not only of $(s, q)$, but also $\alpha$, the angle of the source-lens trajectory relative to the planet-star axis. The narrowness of the boundary reflects that detection is almost independent of $\alpha$. See Batista et al. (2009) and Yee et al. (2009) for concrete illustrations.

It is also striking that the slopes $\eta \sim 0.32$ and $\eta \sim 0.35$ are nearly the same for the two diagrams, leading to the conjecture that $\eta$ is very nearly constant for well-monitored high-magnification events. Indeed, of the 43 events analyzed by Albrow et al. (2001) and Gaudi et al. (2002), two are relatively high mag $\left(A_{\max }>100\right)$ and both have the same triangular appearance and very similar slope $\eta$, as does the extreme $A_{\max }=3000$ event OGLE-BLG-2004-343 with simulated coverage analyzed by Dong et al. (2006). If truly generic, this would mean that high-mag event sensitivities have an extremely simple triangular form characterized by a single parameter, $q_{\min }$.

Moreover, while $q_{\min }$, i.e., the depth of the triangles in Figures 2 and 3, obviously depends on the intensity, quality, and uniformity of coverage, one expects the fundamental scaling to be

$$
q_{\min }=\xi A_{\max }^{-1},
$$

where $\xi$ is a parameter that depends on the data quality, etc. The reason for this expected scaling is that the size of the central caustic is proportional to $q$, and $A_{\max }^{-1}$ measures how closely the source probes the center, which is roughly the maximum of the impact parameter $u_{0}$ and the source size $\rho$ (Han \& Kim 2009; Batista et al. 2009).

Hence, armed with an empirical estimate of $\xi$, one can quickly gage the sensitivity of one event or an ensemble of events to planets, which is quite useful both to guide observations and as a check on "black-box" simulations of event sensitivity. Indeed as we will show below, one can approximately "read off" the frequency of planets by just counting the number detected and the number of high-mag events surveyed.

Based on this handful of published analyses, we estimated $\xi \sim 1 / 70$ for a $\Delta \chi^{2}=500$ threshold. Since these analyses (naturally) focused on events with better-than-average coverage, we estimate that $\xi \sim 1 / 50$ is more appropriate for a sample such as ours. 


\subsection{Analytic Estimate From Triangles}

We now assume that planets are distributed uniformly in $\log s$ (Öpik's law (Opik 1924)) in the neighborhood of the Einstein ring. ${ }^{86}$ We will show further below that this assumption is consistent with current microlensing data. Then, assuming $\eta=0.32$ to be universal, we have

$$
P_{i}(q) d \log q=2 \eta f_{i}(q) \log \frac{q}{q_{\min , i}} \Theta\left(q-q_{\min , i}\right) d \log q,
$$

where $f(q)$ is the number of planets per dex of projected separation per dex of mass ratio and $\Theta$ is a step function. The expected number of planets detected in high-mag events is then just the sum of Equation (4) over all high-mag events with good coverage

$$
\frac{d N_{\mathrm{pl}}}{d \log q}=\sum_{i=1}^{N_{\mathrm{ev}}} P_{i}(q) .
$$

Figure 1 demonstrates that the $\mu \mathrm{FUN}$ sample is uniform for $0<A_{\max }^{-1}<\epsilon$, where $\epsilon=0.005$. Hence, we can turn this sum into an integral,

$$
\begin{aligned}
& \frac{d N_{\mathrm{pl}}}{d \log q}=\sum_{i=1}^{N_{\mathrm{ev}}} P_{i}(q) \rightarrow \frac{N_{\mathrm{ev}}}{\epsilon} \int_{0}^{\epsilon} P_{A_{\max }}(q) d A_{\mathrm{max}}^{-1} \\
& \left.\left.=2 \eta f(q) \frac{N_{\mathrm{ev}}}{\epsilon} \int_{0}^{\epsilon} \log \left[q /\left(\xi / A_{\max }\right)\right] \Theta\left(q-\xi / A_{\max }\right)\right]\right) d A_{\max }^{-1} \\
& =\frac{2 \eta}{\xi} f(q) \frac{N_{\mathrm{ev}}}{\epsilon} \int_{0}^{\xi \epsilon} \log \left(q / q_{\min }\right) \Theta\left(q-q_{\min }\right) d q_{\min } \\
& =\frac{2 \eta}{\xi \ln 10} f(q) \frac{N_{\mathrm{ev}}}{\epsilon} \int_{0}^{\min (q, \xi \epsilon)} \ln \left(q / q_{\min }\right) d q_{\min },
\end{aligned}
$$

which may be evaluated,

$$
\begin{gathered}
\frac{d N_{\mathrm{pl}}}{d \log q}=\frac{2 \eta N_{\mathrm{ev}}}{\ln 10} g(q) f(q), \\
g(q)=\frac{q}{q_{\mathrm{thr}}} \quad(q<\xi \epsilon) ; \quad g(q)=1+\ln \frac{q}{q_{\mathrm{thr}}} \quad(q>\xi \epsilon),
\end{gathered}
$$

where $q_{\mathrm{thr}} \equiv \xi \epsilon$. Below this threshold, detection efficiency falls linearly with $q$. Above the threshold, it rises logarithmically with $q$. Note that the appearance of "In 10" in these formulae is an artifact of our having chosen to express the density of planets in units of dex of separation, rather than the "natural unit" of an $e$-folding.

Both the break at $q_{\text {thr }}$ in the normalized survey sensitivity $g(q)$ and the functional forms of $g(q)$ on either side of this break are easily understood from the triangular form of the individual-event sensitivity diagrams. For $q>q_{\mathrm{thr}}$, all $N_{\mathrm{ev}}$ events contribute sensitivity. If we compare two mass ratios, $\log q$ and $\log q+d \log q$, the latter is sensitive to a $\log$-separation interval on the triangle that is larger by exactly $2 \eta d \log q$ for each individual event, so the sensitivity of the ensemble of events is simply $2 \eta \log q+$ const. On the other hand, for $q<q_{\mathrm{thr}}$, only a fraction $q / q_{\mathrm{thr}}$ of the events contribute, which breaks the logarithmic form of $g(q)$.

\footnotetext{
86 If in fact planets are distributed $d N / d \log s \propto s^{p}$, then Equation (4) is in error by $\sinh x / x$ with $x=\eta p \ln q / q_{\min }$. For $\eta=0.32, p=0.4$ and $q / q_{\min }=100$, this is still only a factor 1.06 .
}

Note that our estimate $\xi=1 / 50$ implies that our $A_{\max }<$ $\epsilon^{-1}=200$ survey has

$$
q_{\mathrm{thr}}=\epsilon \xi=10^{-4}
$$

i.e., twice the mass ratio of Neptune. We discuss the implications of this threshold in Section 6.

\section{NUMERICAL EVALUATION}

\subsection{Sensitivities for the Full Sample}

To more accurately determine the sensitivity of our survey and to infer the frequency of planets, we carry out a detailed sensitivity analysis of all 13 of the $A_{\max }>200$ events in Table 1 (except the two that were already done). We use the method of Rhie et al. (2000) (outlined in Section 3.2) except that we take full account of finite-source effects (Dong et al. 2006), which are much more important for the present sample of events because of their higher magnification. For the last three events above the cut in Table $1, \theta_{\mathrm{E}}$ (and hence $\rho \equiv \theta_{*} / \theta_{\mathrm{E}}$ ) is not well measured. For these, we follow the procedure of Gaudi et al. (2002) and adopt $\rho=\theta_{*} /\left(\mu_{\text {typ }} t_{\mathrm{E}}\right)$, where $\theta_{*}$ is determined from the instrumental color-magnitude diagram in the standard way (Yoo et al. 2004), $t_{\mathrm{E}}$ is the measured Einstein timescale,

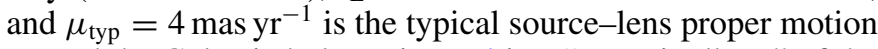
toward the Galactic bulge. Figure 4 is a "portrait album" of the resulting triangle diagrams (only one side shown to conserve space) and Figure 5 shows the integrated sensitivity of each event as a function of mass ratio $q$. That is, it is the integral of the sensitivity (in Figures 2-4) over horizontal slices. Hence, if the sensitivity were truly a triangle, the curves in Figure 5 would be perfectly straight lines, with slope $2 \eta$ (illustrated by the bold black line segment) and $x$-intercepts at $q_{\min }$. Most of the curves do have this behavior over the range $-4 \lesssim \log q \lesssim-2$, which is the main range of sensitivity of this technique and also where the planets in Table 2 are located. Moreover, the inferred intercepts of the straight-line portion of these curves do generally reach to lower mass ratio $q_{\min }$ for higher $A_{\max }$ events, although with considerable scatter. However, while some events (like OGLE-2008-BLG-279) are almost perfectly straight down to zero, others (like OGLE-2005-BLG-169 and OGLE-2006BLG-109) show a pronounced flattening toward lower mass ratios. There are two reasons for this. Events like OGLE-2005BLG-169 have non-uniform coverage over peak, which makes detectability a strong function of angle. The contours in the triangle diagram separate, so that while the $50 \%$ sensitivity contour is fairly straight, there is still substantial sensitivity below $q_{\mathrm{min}}$, which is defined by where the two $50 \%$ contours meet, creating a long tail of sensitivity below this threshold. Events like OGLE-2006-BLG-109 have very small source size relative to impact parameter, $\rho / u_{0} \ll 1$, which enables detection of small mass-ratio planets that are very close to the Einstein ring because the relatively large, but very weak, caustics of these planets are then not "washed out" as they would be for larger $\rho$ (Bennett \& Rhie 1996). Hence, the entire "triangle" has a curved appearance, although the contours are tightly packed together.

The black bold dashed curve in Figure 5 is the combined sensitivity, i.e., the sum of the sensitivities for all 13 events (divided by 10 , so it fits on the same plot), which we call $G(q)$.

The curves in Figure 5 allow us to compare the observed log projected separation $s$, with the maximum detectable separation (see Table 2). Because some planets suffer from the $s \leftrightarrow s^{-1}$ degeneracy, we only show the absolute value of $\log s$. The 


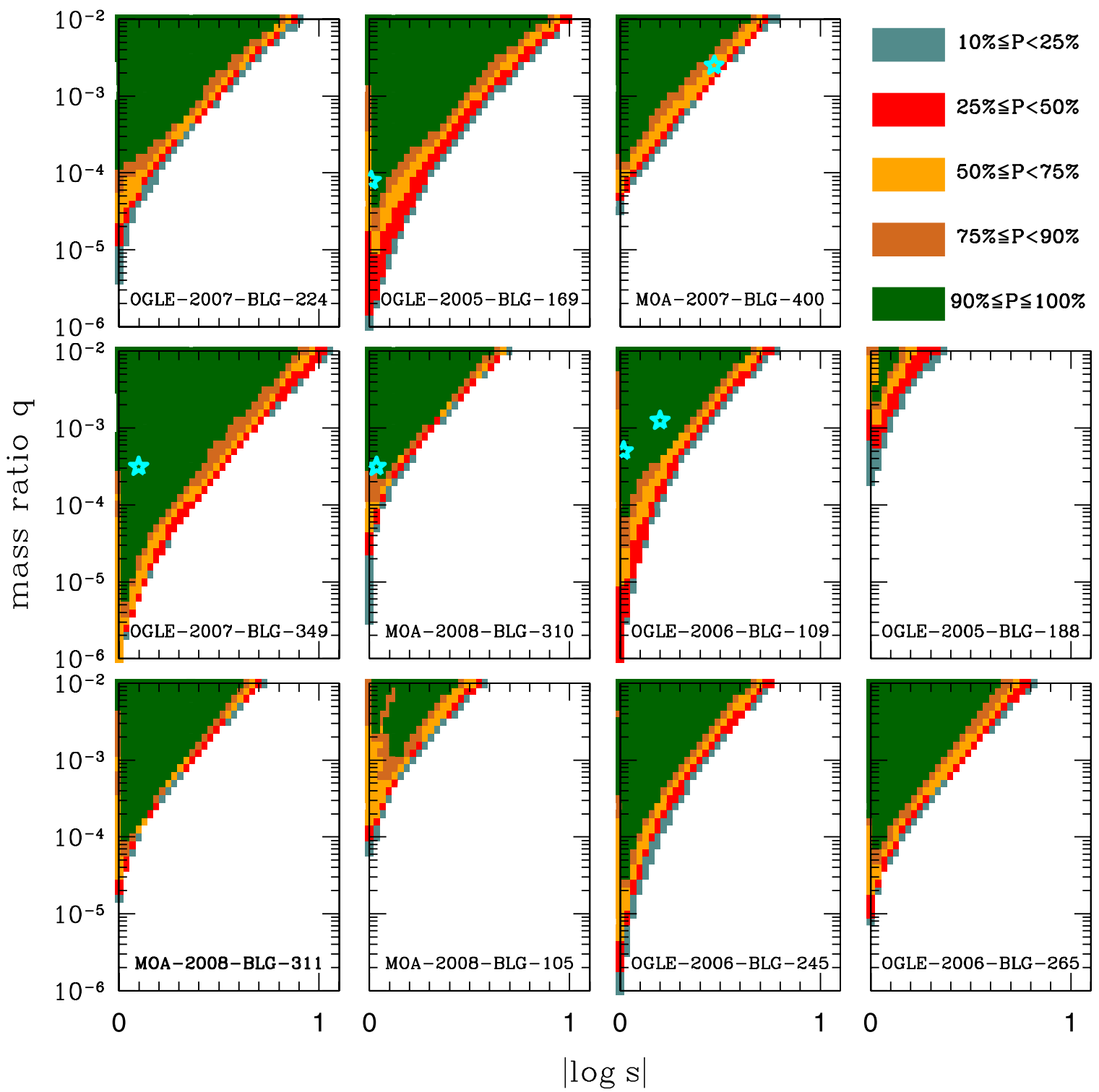

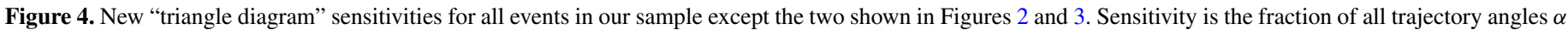

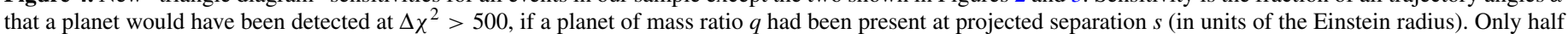

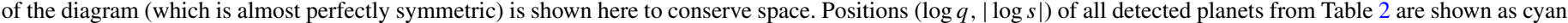
stars.

cumulative distribution of the ratios of these quantities is shown in Figure 6. The separations are consistent with being uniform in $\log s$, with Kolmogorov-Smirnov (K-S) probability of $20 \%$. (Moreover, in general, the high-magnification events with the greatest values of $|\log s|$-and so the greatest potential leverage for probing the distribution as a function of $s$-are also the most severely affected by the $s \leftrightarrow s^{-1}$ degeneracy. This applies, for example, to MOA-2007-BLG-400Lb. Thus, the dependence on $s$ will be much better explored using "planetary caustics" in low-magnification events, for which the $s \leftrightarrow s^{-1}$ degeneracy is easily resolved; Gould \& Loeb 1992; Gaudi \& Gould 1997).

\subsection{Masses of Host Stars}

Now, $f(q)$ may in principle be a function of the host mass $M$ (and perhaps other variables as well). With only six detections, we are obviously in no position to subdivide our sample. Nevertheless, it is important to assess what host mass range we are actually probing. There do exist mass estimates or limits for all five hosts of the planets that have been detected, and there are also mass estimates for 5 of the 8 lensing stars in the sample for which no planet was detected, which are given in Table 1 together with the method of estimation. For five of the events, both the angular Einstein radius $\theta_{\mathrm{E}}$ and the "microlens parallax" $\pi_{\mathrm{E}}$ are measured, which together permit a mass measurement $M=\theta_{\mathrm{E}} / \kappa \pi_{\mathrm{E}}$, where $\kappa \equiv 4 G /\left(c^{2} \mathrm{AU}\right) \sim 8.1$ mas $M_{\odot}^{-1}$ (Gould 2000b). The measurement for OGLE-2007-BLG-349 is preliminary (S. Dong et al., in preparation) but the others are secure. For four of the events, there is a measurement of $\theta_{\mathrm{E}}=\sqrt{\kappa M \pi_{\text {rel }}}$, where $\pi_{\text {rel }}$ is the source-lens relative parallax, but not $\pi_{\mathrm{E}}$. This measurement of the product of $M$ and $\pi_{\text {rel }}$ combined with the lens-source relative proper motion $\mu=\theta_{\mathrm{E}} / t_{\mathrm{E}}$ and a Galactic model (GM) permit a Bayesian estimate of $M$. Finally, there are two events for which adaptive optics (AO) observations provide information on the host. For OGLE-2006-BLG-109, AO resolution of the host confirms the microlensing mass determination from $\theta_{\mathrm{E}}$ and $\pi_{\mathrm{E}}$. For MOA2008-BLG-310, AO observations detect excess light (not due 


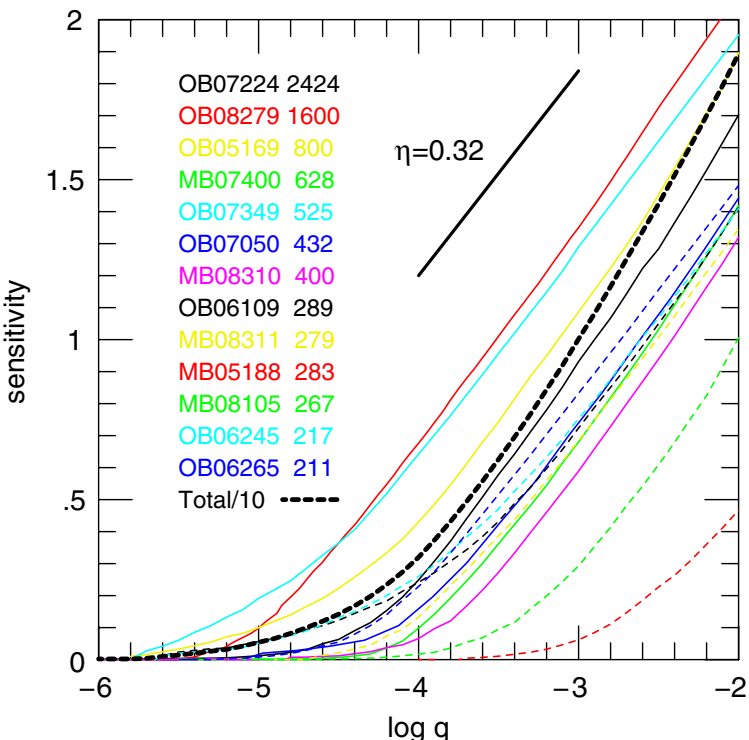

Figure 5. Calculated sensitives for each of the 13 microlensing events. These are integrals over horizontal cuts (at fixed $q$ ) in Figures 2-4. Events are shown in "rainbow order" according to magnification, with the first seven in solid lines and the last six in dashed lines. The bold dashed black curve represents the total sensitivity of the sample (divided by 10).

to the source) but it is not known whether this excess is due to the lens or another star. So only an upper limit on the mass is obtained.

Seven of these 10 measurements are in the range of middle $\mathrm{M}$ to middle $\mathrm{K}$ stars, while one is a brown dwarf and another is likely to be a late $\mathrm{M}$ dwarf. They cover a fairly broad range approximately centered on $0.5 M_{\odot}$, with a tail toward lower mass. (Note that this range includes white dwarfs as well as main-sequence stars.) Of course, there are also three lenses in the $A_{\max }>200$ sample for which there is no mass measurement or estimate. These have timescales $t_{\mathrm{E}}$ of 10, 26, and 59 days, which are quite typical of microlensing events. If these are otherwise typical events, then the lenses probably lie mostly in the Galactic bulge (Kiraga \& Paczyński 1994), in which case their mean mass is roughly $0.4 M_{\odot}$ (Gould 2000a). Given that this is a minority of the sample, that the information about this minority is far less secure, and that the difference from the sample with harder information is not very large, we adopt

$$
M \sim 0.5 M_{\odot}
$$

for the typical mass of the sample. However, we note that the implications discussed in Section 6 would not be greatly affected if we had adopted $M \sim 0.4 M_{\odot}$.

\subsection{Likelihood Analysis}

To evaluate the mass-ratio distribution function $f(q)=A q^{n}$, we maximize the likelihood:

$$
L=-N_{\exp }+\sum_{i=1}^{N_{\mathrm{obs}}} \ln G\left(q_{i}\right) f\left(q_{i}\right) ; \quad N_{\exp }=\int_{q_{-}}^{q_{+}} d q G(q) f(q)
$$

and find

$$
\begin{aligned}
f(q) & =\frac{d N_{\mathrm{ev}}}{d \log q d \log s} \\
& =(0.36 \pm 0.15) \times\left(\frac{q}{5 \times 10^{-4}}\right)^{-0.60 \pm 0.20} \mathrm{dex}^{-2} .
\end{aligned}
$$

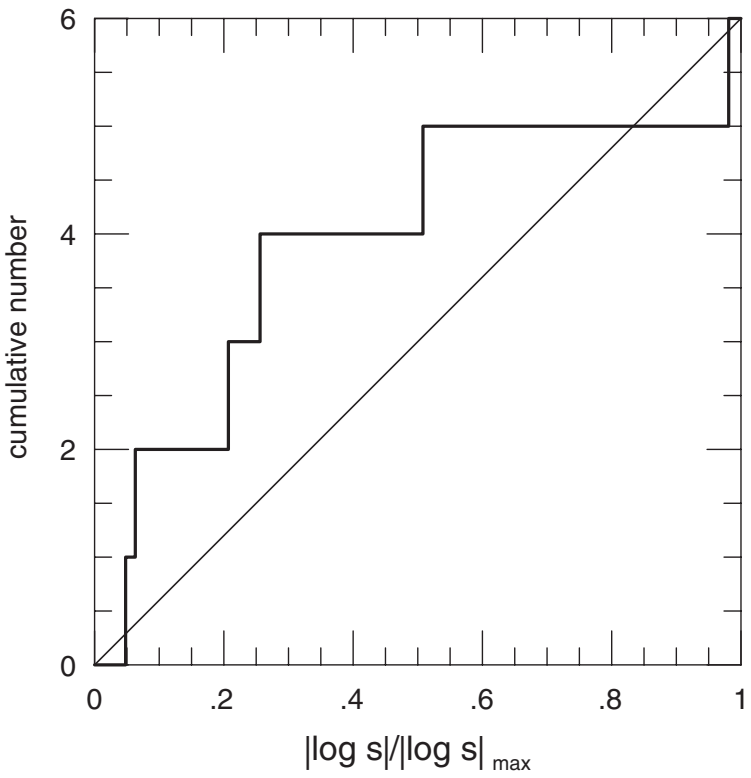

Figure 6. Cumulative distribution of the (absolute value of the log of the) measured projected separation, $|\log s|$, in units of the maximum value of this parameter, derived in Section 5.1. The observed distribution is consistent with planets being distributed uniformly in $\log s$, with Kolmogorov-Smirnov probability of $20 \%$.

However, as we now argue, while the normalization of Equation (12) is robust, the slope is not.

In Section 3.2, we summarized why there must be some boundaries $q_{ \pm}$beyond which the experiment is seriously degraded, but argued that there is no "impartial algorithm" for deciding exactly where those boundaries should be. We find that if we vary $q_{+}$between -2.3 and -2 ., and we vary $q_{-}$between -5 and -4.5 , that the normalization in Equation (12) varies by only $\pm 10 \%$, which is much smaller than the statistical errors. However, the power-law index varies between -0.6 and -0.2 . If we had a much larger sample of planets, then we could set the boundaries at various places within the range of our detections, thereby simultaneously reducing both the number of detections and $N_{\text {exp }}$ in Equation (11). For an infinite sample, such a procedure should lead to no variation in either slope or normalization. For a finite sample, the variation would provide an estimate of the error in these quantities due to the uncertainty in knowledge of these boundaries. However, when we apply this procedure to our small sample, we find quite wild variations, implying that we cannot derive a reliable slope from these data.

In Section 3.2, we mentioned that the threshold value $\Delta \chi^{2}>$ 500 also had some intrinsic uncertainty. However, in this case the effect is very small. For example, if we decrease the threshold to $\Delta \chi^{2}>350$, then the normalization in Equation (12) decreases by only $7 \%$, much less than the Poisson error. Given that the normalization in Equation (12) is robust but the slope is not, we give our final result as

$\frac{d N_{\mathrm{ev}}}{d \log q d \log s}=(0.36 \pm 0.15) \mathrm{dex}^{-2} \quad$ at $q \sim 5 \times 10^{-4}$.

Figure 7 summarizes the principal inputs to the modeling. The top panel shows the cumulative distribution of the detections. The bottom panel shows the sensitivity of the survey as function of planet mass, both the analytic approximation derived in Section 4.2 and the numerical determination derived in Section 5.1. These hardly differ. 

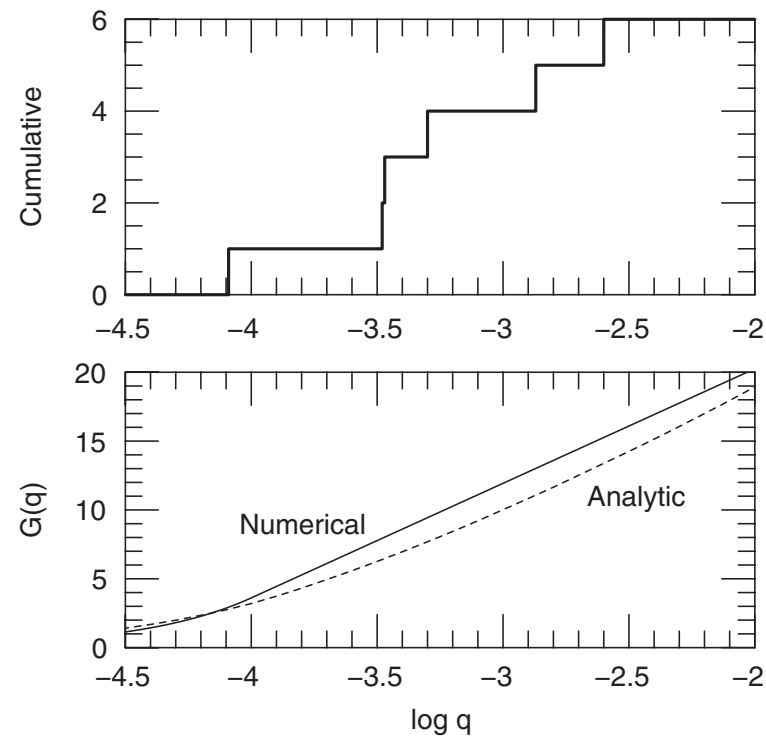

Figure 7. Principal inputs to the modeling. Top panel: cumulative distribution of planet detections in four years of intensive monitoring of high-magnification events. Bottom panel: sensitivity of the survey as a function of planet/star mass ratio $q$, both the analytic approximation derived in Section 4.2 and the numerical determination derived in Section 5.1 and shown in Figure 5. These hardly differ.

The density given in Equation (13) can be obtained by a very simple argument. The "triangle" for each event has sensitivity to $(1 / 2$ base $\times$ height $)=\eta\left[\log \left(0.01 A_{\max } / \xi\right)\right]^{2} \sim$ $1.7\left[1+0.42 \log \left(A_{\max } / 400\right)\right]^{2} \mathrm{dex}^{2}$ of planet parameter space. We observed $N=13$ events and found six planets, so $6 /(13 \times 1.7) \sim 0.27 \mathrm{dex}^{-2}$, i.e., correct within the statistical error.

\section{DISCUSSION}

We have presented the first measurement of the absolute frequency of planets beyond the snow line over the mass-ratio range $-4.5 \lesssim \log q \lesssim-2$. The resulting planet frequency, Equation (13), can be understood directly from the data and the "triangle" sensitivity diagrams. The result is applicable to a range of host masses centered near $M \sim 0.5 M_{\odot}$. The distribution is consistent with being flat in log-projected separation $s$, with sensitivity spanning a range $|\log s| \lesssim$ $(1 / 3)(\log q-4.3)$, and peak sensitivity at projected separation $r_{\perp} \sim 2.5 \mathrm{AU}\left(M_{\text {host }} / 0.5 M_{\odot}\right)^{1 / 2}$.

\subsection{Comparison with Previous Microlensing Results}

Gould et al. (2006) had earlier concluded that "cool Neptunes are common" based on one of the planets analyzed here (OGLE-2005-BLG-169Lb) and another planet with similar mass ratio, OGLE-2005-BLG-390Lb (Beaulieu et al. 2006), which had been detected through another channel: follow-up observations of low-magnification events. OGLE-2005-BLG$390 \mathrm{Lb}$ was actually recognized as a possible planetary event during the planetary deviation, but detailed review of these communications and their impact on the observing schedule shows that this "feedback" was not critical to robust detection. Moreover, at that time there had been no other detections through this channel, so the Beaulieu et al. (2006) detection could also be treated as a "controlled experiment." These could then be combined to obtain an absolute rate for "cool Neptunes," albeit with large errors. However, with only two detections, Gould et al. (2006) were not able to specify the mass range of "cool
Neptunes" and hence were not able to express their results in units of $\mathrm{dex}^{-2}$ as we have done here. If we nevertheless, somewhat arbitrarily, say that the Beaulieu et al. (2006) and Gould et al. (2006) result applies to $1 \mathrm{dex}$ in $\log q$, centered at the $q=8 \times 10^{-5}$ of their two detections, and adopt their $0.4 \mathrm{dex}$ interval in $\log s$, their estimated density of cool Neptunes can be translated to $0.95_{-0.55}^{+0.77} \mathrm{dex}^{-2}(1 \sigma)$. To make a fair comparison with Equation (13), it is necessary to adopt some slope for the mass function in order to account for the factor $\sim 6$ difference in mass. If we adopt the Sumi et al. (2010) slope of $n=-0.68$ from microlensing, then our prediction for this mass range would be $1.25 \mathrm{dex}^{-2}$. If we adopt the Cumming et al. (2008) slope of $n=-0.31$ from RV, it would be $0.64 \mathrm{dex}^{-2}$. Either way, these are consistent.

Sumi et al. (2010) analyzed all 10 published microlensing planets, including the five that we analyze here. They approximated the sensitivity functions of this heterogeneous sample by a single power law $\left(\propto q^{m}, m=0.6 \pm 0.1\right)$ and derived a power-law mass-ratio distribution $d N_{\mathrm{pl}} / d \log q \sim q^{n}$, $n=-0.68 \pm 0.2$. Since we are unable to derive a slope from our analysis, and they do not derive a normalization, there can be no direct comparison of results.

\subsection{Comparison with Radial Velocity Results}

Based on an analysis of RV planets, Cumming et al. (2008) derive a normalization of $0.029 \mathrm{dex}^{-2}$, a factor $12 \pm 5$ smaller than the one found here. A factor $\left(5 \times 10^{-4} / 1.66 \times 10^{-3}\right)^{-0.31}=$ 1.5 of this difference is due to the fact that they normalize at higher mass ratio. The remaining factor $8 \pm 3$ difference is most likely due to the different star-planet separations probed by current microlensing and RV experiments. The Cumming et al. (2008) study targets stars with periods of 2-2000 days, corresponding to a mean semimajor axis of $a=0.31 \mathrm{AU}$. Microlensing probes a factor $\sim 3$ beyond the snow line (Figure 8 from Sumi et al. 2010) ${ }^{87}$ To make contact between microlensing observations of primarily lower-mass stars with RV observations of typically solar-type stars, we should consider planets in similar physical conditions, which we choose to normalize by the snow line. That is, we should compare to G-star planets at 3 "snow-line radii," i.e., $a \sim 8$ AU. Hence, the inferred slope between the RV and microlensing measurements is $d \ln N / d \ln a=\log (8 \pm 3) / \log (8 / 0.31)=0.64 \pm 0.16$, which is consistent (at $1.1 \sigma$ ) with the slope of $d \ln N / d \ln a=$ $0.39 \pm 0.15$ derived by Cumming et al. (2008) for RV stars within their period range. Thus, simple extrapolation of the RV density profile derived from planets thought to have migrated large distances, adequately predicts the microlensing results based on planets beyond the snow line that are believed to have migrated much less (see Figure 8). Figure 9 compares microlensing and RV detections as a function of mass ratio $q$.

\subsection{Prospects for Sensitivity to Very Low Mass Planets}

Equation (8) and Figure 5 show a break in sensitivity at $q_{\mathrm{thr}} \simeq 10^{-4}$. For a power-law mass-ratio distribution, the ratio

\footnotetext{
87 Just as RV measurements respond to projected stellar velocities, and so measure $m \sin i$ of the planet which is always less than or equal to the planet mass $m$, so microlensing observations measure the projected separation $s$, which for circular orbits is related to the semimajor axis by $R_{\mathrm{E}} s=a \sin \gamma$ where $\gamma$ is the angle between the star-planet axis and the line of sight. The statistical distribution $\sin \gamma$ is exactly the same as for $\sin i$ in RV. Hence, except for rare cases when the orbit is constrained by higher-order effects (Dong et al. 2009a; Bennett et al. 2010), $a$ must be statistically estimated from $s$ (and $R_{\mathrm{E}}$ ), which is what is done in Figure 8 of Sumi et al. (2010).
} 


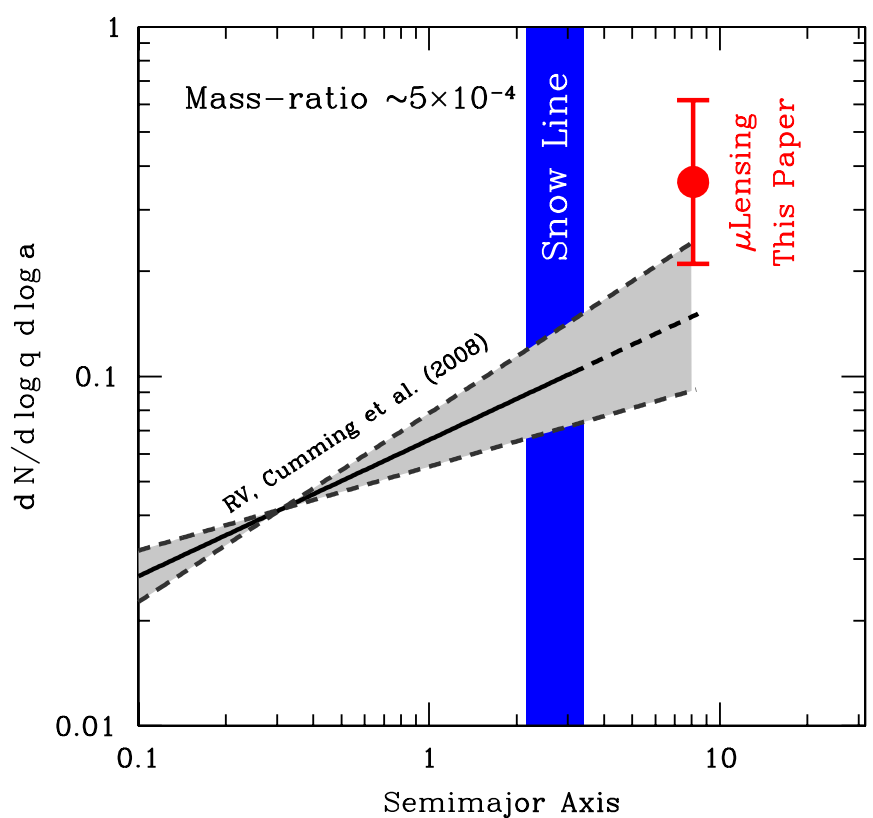

Figure 8. Planet frequencies determined from microlensing (this paper) and RV (Cumming et al. 2008) at different semimajor axes. The RV result is scaled to mass ratio $q=5 \times 10^{-4}$, using the $\mathrm{RV}$-derived slope, $n=-0.31$. In order to take account of the different host star masses $\left(M \sim 1 M_{\odot}\right.$ for RV, $M \sim 0.5 M_{\odot}$ for microlensing) we have placed the microlensing point at $8 \mathrm{AU}$, i.e., three times the solar-system "snow line" distance. This is because microlensing planets are typically detected at three times the distance of their own systems' snow line (which is of course much closer than $8 \mathrm{AU}$ ).

(A color version of this figure is available in the online journal.)

of planets expected above and below this threshold is

$$
\frac{N_{\mathrm{pl}}\left(q>q_{\mathrm{thr}}\right)}{N_{\mathrm{pl}}\left(q<q_{\mathrm{thr}}\right)}=\frac{n+1}{n}\left[z^{n} \ln (z)+\left(\frac{n-1}{n}\right)\left(z^{n}-1\right)\right],
$$

where $z \equiv 0.01 / q_{\mathrm{thr}}$. This ratio is an extremely strong function of the adopted slope of the mass function, $n$. For $n=-0.68$ (Sumi et al. 2010), it is 1.0, whereas for $n=-0.31$ (Cumming et al. 2008), it is 4.7. The fraction of planets within the lower domain that lies below $q$ is simply $q^{n+1}$. Hence, while several authors have shown that individual planets at or near Earth mass ratio are detectable in high-magnification events (Abe et al. 2004; Dong et al. 2006; Yee et al. 2009; Batista et al. 2009), the actual rate of detection will be strongly influenced by the actual value of $n$. As discussed in Section 3.2, probing to lower masses will require technical advances to robustly identify low-mass planets in the presence of higher-mass planets. But it will also require increasing the number of events that are monitored.

There is some potential to do this. First, as shown in Section 5, only about half of events that are announced by search teams are intensively monitored. Hence, there is room to double the rate by more aggressive monitoring. This will be aided by inauguration of OGLE-IV, which will have much higher time sampling and so will permit more accurate prediction of high-mag events. Second, it is possible that the more intensive OGLE-IV survey will increase the underlying sample of high-mag events. Finally, systematic analysis of high-mag events could bring down the effective $\Delta \chi^{2}$ threshold from 500 to, say, 200, which would decrease $\xi$ (and so $q_{\text {thr }}$ ) by a factor $(500 / 200)^{2 / 3} \sim 1.8$. This would bring only a modest (logarithmic) increase in the sensitivity in the range $q>q_{\text {thr }}$ but would aid linearly for $q<q_{\text {thr }}$.

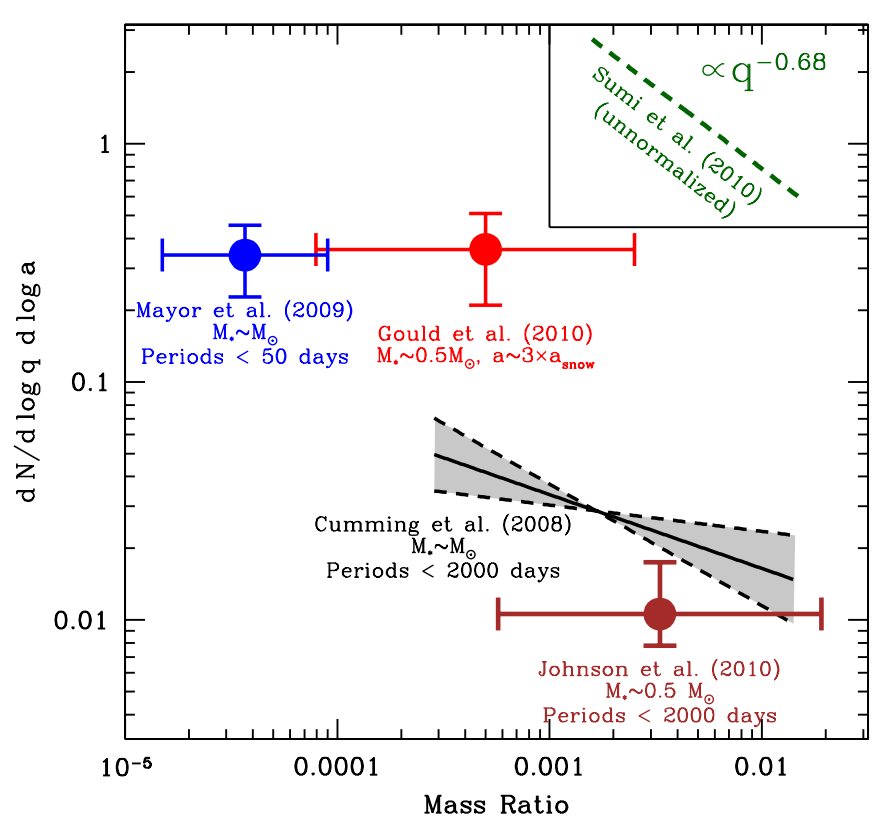

Figure 9. Planet frequencies as a function of mass ratio for microlensing (this paper) and RV (Cumming et al. 2008; Mayor et al. 2009; Johnson et al. 2010) detections. As illustrated in Figure 8, the Cumming et al. (2008) RV sample and the microlensing sample are consistent with each other (despite different frequencies in this figure) because they are at different distances. The Cumming et al. (2008) and Mayor et al. (2009) RV samples are directly comparable on this diagram because both are $\mathrm{G}$ stars, and they are consistent. However, there is some tension between the Johnson et al. (2010) RV measurement and the microlensing measurement, since both are similar type stars. This is because Cumming et al. (2008) and Johnson et al. (2010) are "inconsistent" with each other, if one assumes that the frequency of planets as a function of mass ratio is independent of host mass, as we have done in arguing for the consistency of microlensing with Cumming et al. (2008). Also shown is the (unnormalized) slope derived from microlensing observations by Sumi et al. (2010).

(A color version of this figure is available in the online journal.)

\subsection{Constraints on Migration Scenarios}

We showed in Section 6.2 that the planet density derived here, $d N_{\mathrm{pl}} / d \log q d \log s=0.36 \mathrm{dex}^{-2}$ is consistent with the density derived from RV studies, if the latter are extrapolated to $\sim 25$ times the semimajor axis where the measurement is made. Regardless of the details of this comparison, the fact that the density of planets beyond the snow line is eight times higher than that at $0.3 \mathrm{AU}$, indicates that most giant planets (that survive migration) do not migrate very far.

Moreover, the fact that the slope found in RV studies at small $s$ adequately predicts the density at large $s$, would seem to imply that whatever is governing the amount of migration is a continuous parameter. That is, it is not the case that there are two classes of planetary systems: those with migration and those without. Rather, all systems have migration, but by a continuously varying amount. This picture would be in accord with the evolving view of the solar system that even though the giant planets are in the general area of their birth "beyond the snow line," they have migrated to a modest degree. However, this "continuous" distribution of migrations may well contain additional substructure. For example, Figure 11 from Cumming et al. (2008) shows a "pile-up" of orbits at $P \sim 1 \mathrm{yr}$, even though the overall distribution is broadly defined by a power law.

\subsection{Comparison to Solar System}

Another interesting point of comparison is to the planet density in the solar system, where there are four planets in the 


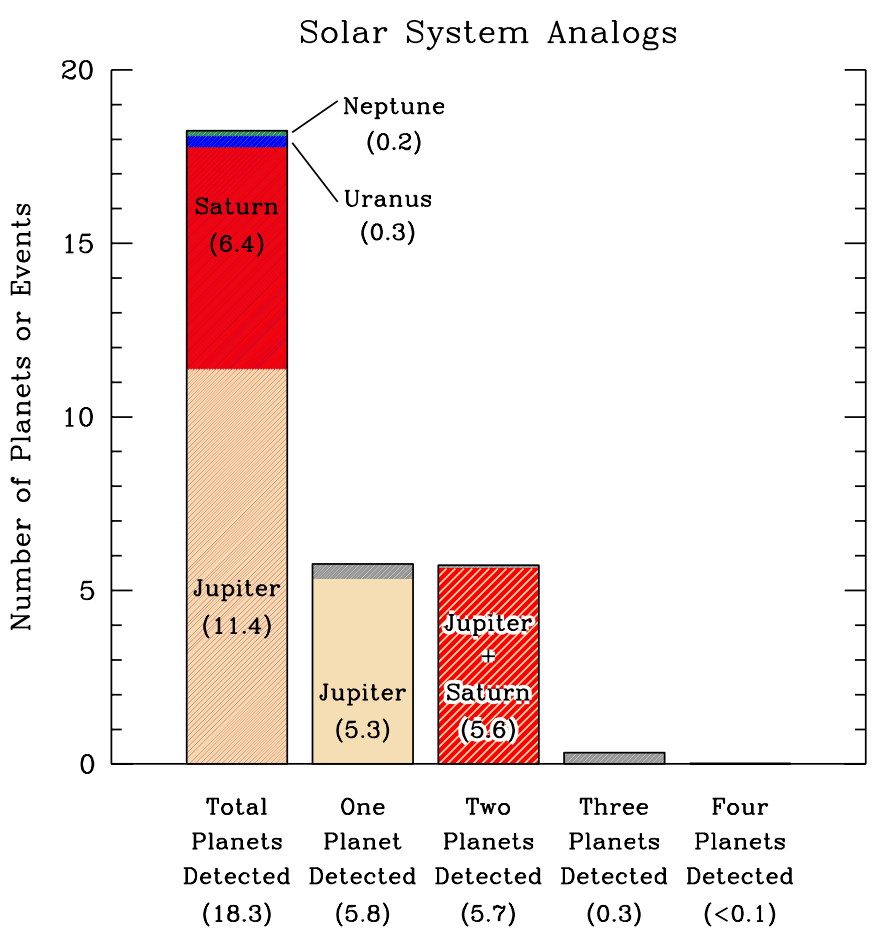

Figure 10. Results of Monte Carlo simulation of the anticipated results of our microlensing survey assuming that every star toward the Galactic bulge had a "scaled solar system," as specified by Equations (15) and (16). A total of 18 planets would have been detected, including six two-planet systems, compared to the actual detections of six planets including one two-planet system. This seems to indicate that the solar system is overdense in planets, especially multiple planets. Also shown are the frequencies of various specific combinations.

mass-separation regime that microlensing currently explores, Jupiter, Saturn, Uranus, and Neptune. How common are "solarsystem analogs," i.e., systems with several giant planets out beyond the snow line?

To address this question, we ask what the result of our study would have been if every microlensed star possessed a "scaled version" of our own solar system in the following sense: four planets with the same planet-to-star mass ratios and same ratios of semimajor axes as the outer solar-system planets, but with the overall scale determined by the "snow line." While there is observational evidence from the asteroid belt that the solarsystem snow line is near $R_{\text {snow, } \odot}=2.7 \mathrm{AU}$ (Morbidelli et al. 2000 ), there is considerable uncertainty on how this scales with stellar mass (Sasselov \& Lecar 2000; Kennedy \& Kenyon 2008). We therefore parameterize this relation by

$$
R_{\text {snow }}(M)=R_{\text {snow }, \odot}\left(\frac{M}{M_{\odot}}\right)^{v}
$$

and consider a range $0.5<v<2$, adopting $v=1$ for our fiducial value. We consider that the typical Einstein radius is $R_{\mathrm{E}}=3.5 \mathrm{AU}\left(M / M_{\odot}\right)^{1 / 2}$ and the typical lensed star is $M=0.5 M_{\odot}$. Then scaling down the semimajor axis of a Jupiter analog so $a_{\text {Jup }- \text { analog }} / R_{\text {snow }}=a_{\text {Jup }} / R_{\text {snow } \odot \odot}$ implies

$$
\frac{a_{\text {Jup }- \text { analog }}}{R_{\mathrm{E}}}=\frac{a_{\text {Jup }}}{3.5 \mathrm{AU}}\left(\frac{M}{M_{\odot}}\right)^{\nu-0.5},
$$

and similarly for the other three planets. We then imagine that these systems are viewed at random orientations, with the individual planets in random phases. From a Monte Carlo simulation, we find that we would then have expected (for our

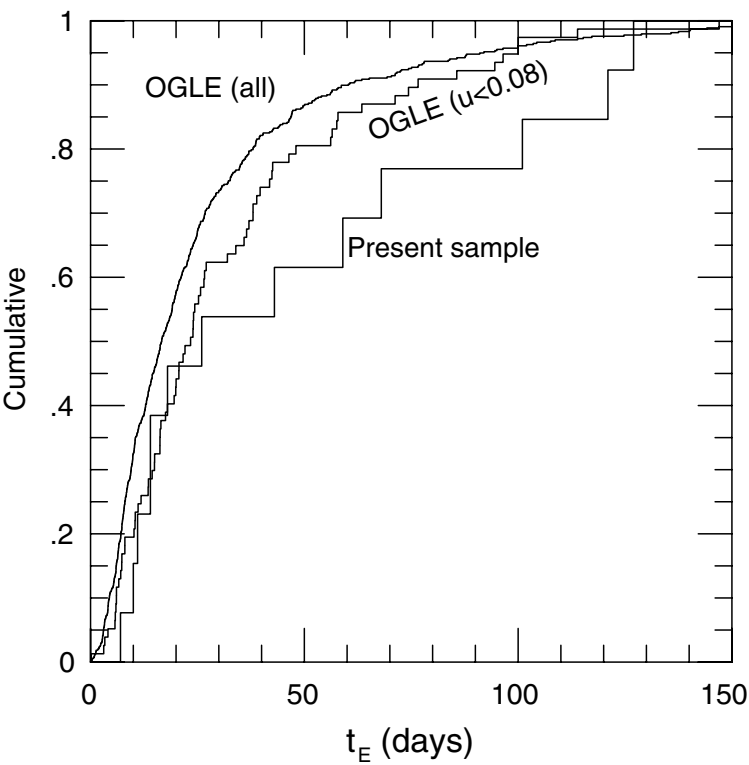

Figure 11. Cumulative timescale $\left(t_{\mathrm{E}}\right)$ distributions of all 537 OGLE events from 2008 that were included in the reanalysis by Cohen et al. (2010) (left), the 77 relatively high-mag $\left(u_{0}<0.08\right)$ events among these (center), and the wellmonitored high-mag sample of 13 events from this paper (right). Each selection appears to result in longer mean timescales. However, a K-S test shows that two right distributions ( 13 well-monitored high mag and 77 relatively high mag) can be drawn from the same distribution at $45 \%$ probability. And the corresponding probability for the all-537 and 13-event samples is 18\%. Even if real, the bias toward long timescale events would not impact the planet frequencies derived in this paper. Rather, they would affect our interpretation of the underlying sample of the host stars that we are probing, primarily by indicating a larger fraction of hosts in the Galactic disk versus the bulge. As such, this bias would have to be accounted for in studies of the Galactic distribution of planets.

fiducial $v=1$ ) to have detected 18.2 planets (11.4 "Jupiters," 6.4 "Saturns," 0.3 "Uranuses," 0.2 "Neptunes") including 6.1 systems with two or more planet detections (see Figure 10). For $1<v<2$, the planet totals and multi-planet detections barely change. However, they fall somewhat for smaller $v$, reaching 15.3 planets and 3.8 two-planet systems at $v=0.5$.

These results compare to six planets including one two-planet system in the actual sample.

Hence, our solar system appears to be three times richer in planets than other stars along the line of sight toward the Galactic bulge. The single detection of a multi-planet system (Gaudi et al. 2008) allows the first estimate of the frequency of stars with "solar-like systems," defined as having multiple giants in the snow zone: $1 / 6$, albeit with large errors.

\subsection{Sample Bias}

A considerable fraction of this paper was devoted to demonstrating that the sample of 13 high-mag events is not biased with respect to the presence (or absence) of planets over the adopted mass-ratio range $-4.5<\log q<-2.0$. Because of its central importance, we briefly recapitulate this argument immediately below. But there is also a second issue as to whether these 13 events represent a "fair sample" of microlenses, i.e., Galactic stars that give rise to microlensing events. As we will explain, this question has no direct implications for the conclusions of this paper, which is why we have not discussed it up to this point. However, it may have implications for future work carried out on this sample and therefore warrants some comment. 
The key point was to design criteria such that for the selected sample, the sensitivity of observations to planets did not depend significantly on the presence or absence of planets. Such a correlation could be introduced if tentative information on the possible presence of a planet caused the observational cadence to increase. Then the (possible) presence of a planet would itself act to increase the $\Delta \chi^{2}$ of detection, perhaps from below to above the adopted threshold. Alternatively, if no planet were detected prior to or during the peak, observations might slacken, causing the sensitivity of the event to fall. Note that both of these potential biases would tend to increase the apparent frequency of planets (by increasing the numerator or decreasing the denominator).

The argument against the first bias (Section 3.3) had two parts. First, for three of the five planet-bearing events, there was no knowledge of the planetary perturbation until well after the event. Second, for the remaining two events, review of the actual observational cadence as well as email traffic showed no substantial deviations from normal monitoring of high-mag events.

The argument against the second bias (Section 3.1 and Figure 1) was that for events with $A_{\max }>200$, the distribution of events meeting the other selection criteria was uniform in $A_{\max }^{-1}$. This is the expected behavior both theoretically and of the full sample of events that are actually observed (whether or not they are monitored for planets).

The requirement for independence of observing cadence from planet presence also sets the upper limit for planet-star mass ratio $q$ to which the experiment is robustly sensitive. Figures 2-4 show that at the adopted limit, $q_{+}=0.01$, most events in the sample are formally sensitive to planets to within a factor 5 (0.7 dex) of the Einstein ring. In fact (as discussed in Section 3.2), the great majority of such events will (prior to perturbations near peak) look like standard, unperturbed, prospective high-mag events, and so will generate "high-mag alerts" in the same way as any other high-mag event. However, for a small fraction, those with separations very close to the Einstein ring, the caustic will be quite large and hence the perturbation will come early, and so may influence (positively or negatively) the decision to take data. At $q=0.01$, this fraction is still small, $\lesssim 20 \%$, which can be seen as follows. About $80 \%$ of detection space lies at $|\log s| \geqslant 20 \% \times 0.7 \mathrm{dex}=0.14 \mathrm{dex}$, at which point the caustic has a half-diameter of $u_{\text {caustic }}=1.8 q=$ 0.018 (Chung et al. 2005). Since high-mag alerts are typically issued at $u \gtrsim 0.04$ (i.e., $A \lesssim 25$ ) when such a caustic does not yet strongly affect the light curve, and since alerts are never canceled because of caustics encountered at $A \gtrsim 50$, fewer than $20 \%$ of perturbed $q=0.01$ events can have their observing cadence affected by the presence of the planet.

As discussed in Section 3.2, the lower-mass-ratio limit is set by the (technological) difficulty of recognizing low-mass-ratio planets in the presence of other planets. That is, our sensitivity calculations accurately reflect our ability to detect planets in single-planet systems. And for two-planet systems in which the two mass ratios are within about an order of magnitude, the perturbations are known to "factor," which means that these same calculations can be used to evaluate sensitivity to a second planet in the presence of the first. However, the effect of combining two-planet perturbations when one has extremely low mass is poorly understood at present. Since 5 of the 13 events had at least one planet, this implies that our sensitivity to lowmass planets is not well understood. We have good confidence in our ability to detect "second planets" at $q>10^{-5}$, but set $q_{-}=10^{-4.5}$ to be conservative.
Finally, we examine the issue of "bias" in the timescale distribution. The first question is "bias with respect to what"? In principle, our densely monitored high-mag sample may be biased with respect to the high-mag events alerted by survey teams, half of which are not densely monitored. And this highmag alert sample might be biased relative to the ensemble of all events alerted by survey groups. Finally, these alerted events might themselves be biased relative to a hypothetical survey that "impartially" recorded all events entering the Einstein ring. And indeed, we expect a bias toward longer timescales at all three levels. Regarding the densely monitored sample, it is easier to recognize events as being high-mag before peak (and so to initiate pre-peak observations) if the event is more drawn out. Regarding survey alerts, high-magnification can bring otherwise undetectable sources above the detection threshold, but this is more likely to yield an alert if the event duration (and so enhanced brightness) lasts longer. The cumulative distributions in Figure 11 are in accord with both these first two expectations, although with at most modest statistical significance. And regarding the third, all microlensing surveys have found that their "detection efficiency" rises monotonically with timescale, until very long timescales are reached.

But what practical implications does this have? For the present study: essentially none. The biases toward longer timescales implies a bias in the underlying lens population. Disk lenses tend to have longer timescales than bulge lenses, and this applies more strongly to relatively nearby disk lenses. Hence, a more timescale-biased sample will have relatively more disk lenses than a less biased sample. But this just means that the planet frequencies derived in this paper apply to whatever disk+bulge sample of lenses is being probed. Whatever the exact breakdown, all of the lenses being probed are much farther from the Sun than the RV samples, so when we compare our results to $\mathrm{RV}$, they are for two different populations. This situation is not altered by the relative fraction of disk stars.

Since the Einstein radius is proportional to the square root of the host mass, more timescale-biased samples will tend to have higher-mass hosts compared to less timescale-biased samples. But this is also of no direct relevance, since we have estimated the typical lens mass based on mass measurements and estimates of lenses in our actual sample (rather than on general theoretical considerations).

As discussed by Janczak et al. (2010), the issue of timescale bias will play an important role if this (or another) sample is used to extract information on the relative frequency of planets orbiting hosts in the disk versus the bulge. For example, of the six planets in our study, three are definitely in the disk (OGLE-2006BLG-109Lb,c and OGLE-2007-BLG-349Lb) and one other is almost certainly in the disk (OGLE-2005-BLG-169Lb), while the remaining two are uncertain. By contrast, the majority of lenses seen toward the bulge, are bulge lenses (Kiraga \& Paczyński 1994). However, no conclusion can be drawn about the relative frequency of bulge and disk planets from this comparison until the timescale (and other) biases are fully analyzed. Since this is not the subject of the present work, we do not attempt such an analysis, but merely alert the reader to the possible presence of these biases.

Work by A.G. was supported in part by NSF grant AST 0757888 and in part by NASA grant 1277721 issued by JPL/ Caltech. Work by S.D. was performed under contract with the California Institute of Technology (Caltech) funded by NASA 
through the Sagan Fellowship Program. This work was supported in part by an allocation of computing time from the Ohio Supercomputer Center. The OGLE project is partially supported by the Polish MNiSW grant N20303032/4275 to AU. The MOA collaboration was supported by the Marsden Fund of New Zealand. The MOA collaboration and a part of authors are supported by the Grant-in-Aid for Scientific Research, JSPS Research fellowships and the Global COE Program "Quest for Fundamental Principles in the Universe" from JSPS and MEXT of Japan. T.S. acknowledges support from grants JSPS18749004, JSPS20740104, and MEXT19015005. The RoboNet project acknowledges support from PPARC (Particle Physics and Astronomy Research Council) and STFC (Science and Technology Facilities Council). C.H. was supported by Creative Research Initiative Program (2009-0081561) of National Research Foundation of Korea. AGY's activity is supported by a Marie Curie IRG grant from the EU, and by the Minerva Foundation, Benoziyo Center for Astrophysics, a research grant from Peter and Patricia Gruber Awards, and the William Z. and Eda Bess Novick New Scientists Fund at the Weizmann Institute. D.P.B. was supported by grants AST-0708890 from the NSF and NNX07AL71G from NASA. Work by S.K. was supported at the Technion by the Kitzman Fellowship and by a grant from the Israel-Niedersachsen collaboration program. Mt Canopus observatory is financially supported by Dr. David Warren.

\section{REFERENCES}

Abe, F., et al. 2004, Science, 305, 1264

Albrow, M., et al. 1998, ApJ, 509, 687

Albrow, M., et al. 2001, ApJ, 556, L113

Alcock, C., et al. 1996, ApJ, 463, L67

Alcock, C., et al. 1997, ApJ, 491, 436

Alcock, C., et al. 2000, ApJ, 541, 270

An, J., et al. 2002, ApJ, 572, 521

Batista, V., et al. 2009, A\&A, 508, 467

Beaulieu, J.-P., et al. 2006, Nature, 439, 437

Bennett, D. P., \& Rhie, S.-H. 1996, ApJ, 472, 660

Bennett, D. P., et al. 2008, ApJ, 684, 663

Bennett, D. P., et al. 2010, ApJ, 713, 837

Bond, I. A., et al. 2002a, MNRAS, 331, L19

Bond, I. A., et al. 2002b, MNRAS, 333, 71

Bond, I. A., et al. 2004, ApJ, 606, L155

Bozza, V. 1999, A\&A, 348, 311

Castro, S., Pogge, R. W., Rich, R. M., DePoy, D. L., \& Gould, A. 2001, ApJ, 548, L197
Chung, S.-J., et al. 2005, ApJ, 630, 535

Cohen, J., et al. 2010, ApJ, 711, L48

Cumming, A., Butler, R. P., Marcy, G. W., Vogt, S. S., Wright, J. T., \& Fischer, D. A. 2008, PASP, 120,531

Dominik, M., et al. 2007, MNRAS, 307, 792

Dominik, M., et al. 2008, Astron. Nachr., 329, 248

Dong, S., et al. 2006, ApJ, 642, 842

Dong, S., et al. 2009a, ApJ, 695, 442

Dong, S., et al. 2009b, ApJ, 698, 1826

Gaudi, B. S., \& Gould, A. 1997, ApJ, 486, 85

Gould, A., \& Loeb, A. 1992, ApJ, 396, 104

Gaudi, B. S., Naber, R. M., \& Sackett, P. D. 1998, ApJ, 502, L33

Gaudi, B. S., \& Sackett, P. D. 2000, ApJ, 528, 56

Gaudi, B. S., et al. 2002, ApJ, 566, 463

Gaudi, B. S., et al. 2008, Science, 319, 927

Gould, A., et al. 2000a, ApJ, 535, 920

Gould, A., et al. 2000b, ApJ, 542, 785

Gould, A., et al. 2006, ApJ, 644, L37

Griest, K., \& Safizadeh, N. 1998, ApJ, 500, 37

Han, C. 2005, ApJ, 629, 1102

Han, C., \& Kim, D. 2009, ApJ, 693, 1835

Horne, K., Tsapras, Y., \& Snodgrass, C. 2009, MNRAS, 396, 2087

Janczak, J., et al. 2010, ApJ, 711, 731

Jaroszyński, J., et al. 2006, Acta Astron., 56, 307

Johnson, J. A., et al. 2010, PASP, 122, 149

Kennedy, G. M., \& Kenyon, S. J. 2008, ApJ, 673, 502

Kiraga, M., \& Paczyński, B. 1994, ApJ, 430, L101

Liebes, S. 1964, Phys. Rev., 133, 835

Mao, S., \& Paczyński, B. 1991, ApJ, 374, L37

Mayor, M., et al. 2009, A\&A, 493, 639

Morbidelli, A., Chambers, J., Lunine, J. I., Petit, J. M., Robert, F., Valsecchi, G. B., \& Cyr, K. E. 2000, Meteorit. Planet. Sci., 35, 1309

Öpik, E. 1924, Pulications de. L'Observatoire Astronomique de l'Université de Tartu, 25, 6

Rattenbury, N. J., Bond, I. A., Skuljan, J., \& Yock, P. C. M. 2002, MNRAS, 335,159

Rhie, S. H., Becker, A. C., Bennett, D. P., Fragile, P. C., Johnson, B. R., King, L. J., Peterson, B. A., \& Quinn, J. 1999, ApJ, 522, 1037

Rhie, S. H., et al. 2000, ApJ, 533, 378

Sasselov, D. D., \& Lecar, M. 2000, ApJ, 528, 995

Snodgrass, C., Horne, K., \& Tsapras, Y. 2004, MNRAS, 351, 967

Sumi, T., et al. 2010, ApJ, 710, 1641

Tsapras, Y., Horne, K., Kane, S., \& Carson, R. 2003, MNRAS, 343, 1131

Tsapras, Y., et al. 2009, Astron. Nachr., 330, 4

Udalski, A. 2003, Acta Astron., 53, 291

Udalski, A., Szymanski, M., Kaluzny, J., Kubiak, M., Mateo, M., Krzeminski, W., \& Paczynski, B. 1994, Acta Astron., 44, 317

Udalski, A., et al. 2005, ApJ, 628, L109

Yee, J. C., et al. 2009, ApJ, 703, 2082

Yoo, J., et al. 2003, ApJ, 616, 1204

Yoo, J., et al. 2004, ApJ, 603, 139 